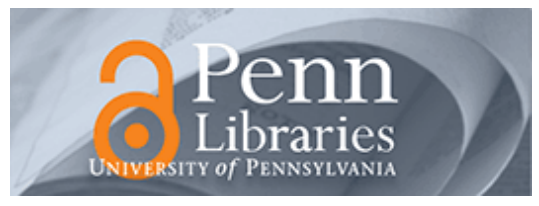

University of Pennsylvania

ScholarlyCommons

Marketing Papers

Wharton Faculty Research

2010

\title{
A Theory of Regular Markov Perfect Equilibria in Dynamic Stochastic Games: Genericity, Stability, and Purification
}

Ulrich Doraszelski

University of Pennsylvania

Juan F. Escobar

Follow this and additional works at: https://repository.upenn.edu/marketing_papers

Part of the Behavioral Economics Commons, Econometrics Commons, and the Marketing Commons

\section{Recommended Citation}

Doraszelski, U., \& Escobar, J. F. (2010). A Theory of Regular Markov Perfect Equilibria in Dynamic Stochastic Games: Genericity, Stability, and Purification. Theoretical Economics, 5369-402. http://dx.doi.org/10.3982/TE632

This paper is posted at ScholarlyCommons. https://repository.upenn.edu/marketing_papers/409

For more information, please contact repository@pobox.upenn.edu. 


\title{
A Theory of Regular Markov Perfect Equilibria in Dynamic Stochastic Games: Genericity, Stability, and Purification
}

\author{
Abstract \\ This paper studies generic properties of Markov perfect equilibria in dynamic stochastic games. We show \\ that almost all dynamic stochastic games have a finite number of locally isolated Markov perfect \\ equilibria. These equilibria are essential and strongly stable. Moreover, they all admit purification. To \\ establish these results, we introduce a notion of regularity for dynamic stochastic games and exploit a \\ simple connection between normal form and dynamic stochastic games.

\section{Keywords} \\ dynamic stochastic games, Markov perfect equilibria, regularity, genericity, finiteness, strong stability, \\ essentiality, purifiability, estimation, computation, repeated games \\ Disciplines \\ Behavioral Economics | Business | Econometrics | Marketing
}




\title{
A theory of regular Markov perfect equilibria in dynamic stochastic games: Genericity, stability, and purification
}

\author{
ULRICH DORASZELSKI \\ Wharton School, University of Pennsylvania \\ JUAN F. ESCOBAR \\ Department of Industrial Engineering, University of Chile
}

\begin{abstract}
This paper studies generic properties of Markov perfect equilibria in dynamic stochastic games. We show that almost all dynamic stochastic games have a finite number of locally isolated Markov perfect equilibria. These equilibria are essential and strongly stable. Moreover, they all admit purification. To establish these results, we introduce a notion of regularity for dynamic stochastic games and exploit a simple connection between normal form and dynamic stochastic games.
\end{abstract}

KeYwords. Dynamic stochastic games, Markov perfect equilibrium, regularity, genericity, finiteness, strong stability, essentiality, purifiability, estimation, computation, repeated games.

JEL CLASSIFICATION. C61, C62, C73.

\section{INTRODUCTION}

Stochastic games are central to the analysis of strategic interactions among forwardlooking players in dynamic environments. Dating back to Shapley (1953), they have a long tradition in economics. Applications of dynamic stochastic games abound and range from public finance (Bernheim and Ray 1989) and political economics (Acemoglu and Robinson 2001) to industrial organization (Bergemann and Valimaki 1996). An especially well known example is the Ericson and Pakes (1995) model of dynamic competition in an oligopolistic industry with investment, entry, and exit that has triggered a large and active literature in industrial organization (see Doraszelski and Pakes 2007 for a survey) and other fields.

While several results in the literature guarantee the existence of Markov perfect equilibria in dynamic stochastic games, to date very little is known about the structure of the

Ulrich Doraszelski: doraszelski@wharton . upenn. edu

Juan F. Escobar: jescobar@dii.uchile.cl

We are greatly indebted to Michaela Draganska, John Duggan, Drew Fudenberg, Dino Gerardi, Hari Govindan, Paul Grieco, Matt Jackson, Ken Judd, Carlos Lever, Jon Levin, Michael Ostrovsky, Ronald Peeters, Martine Quinzii, Tomás Rodriguez, Ilya Segal, Andy Skrzypacz, Bob Wilson, the Editor Ed Green, three anonymous referees, and seminar participants at UC Davis, Rochester, Stanford, SED (2008), and Games (2008) for comments and suggestions. Doraszelski gratefully acknowledges the hospitality of the Hoover Institution during the academic year 2001/2007 and financial support from the National Science Foundation under Grant 0615615. Escobar acknowledges the support of Fondecyt Project 11090166.

Copyright (C) 2010 Ulrich Doraszelski and Juan F. Escobar. Licensed under the Creative Commons Attribution-NonCommercial License 3.0. Available at http: //econtheory . org.

DOI: 10.3982/TE632 
equilibrium set in a dynamic environment. This paucity of knowledge sharply contrasts with normal form games, where a large body of research is devoted to characterizing the equilibrium set and the properties of its members.

The goal of this paper is to help fill this void by developing a theory of regular Markov perfect equilibria in discrete-time, infinite-horizon dynamic stochastic games with a finite number of states and actions. We introduce a suitable regularity notion and show that regularity is a generic property of Markov perfect equilibria. More formally, we identify a dynamic stochastic game with its period payoffs and show that the set of games having Markov perfect equilibria that all are regular is open and has full Lebesgue measure. While regularity is a purely mathematical concept, it paves the way to a number of economically meaningful properties. An immediate consequence of the fact that all Markov perfect equilibria of almost all dynamic stochastic games are regular is that almost all games have a finite number of Markov perfect equilibria that are locally isolated. With some further work, it can be shown that these equilibria are essential and strongly stable, and are therefore robust to slight changes in payoffs. Finally, they all admit purification and can, therefore, be obtained as limits of equilibria of dynamic stochastic games of incomplete information as random payoff fluctuations become vanishingly small. In sum, this paper shows how to extend several of the most fundamental results, including genericity (Harsanyi 1973a), stability (Wu and Jiang 1962, Kojima et al. 1985), and purifiability (Harsanyi 1973b), of the by now standard theory of regular Nash equilibria in normal form games from static to dynamic environments.

Our starting point is the observation that, holding fixed the value of continued play, the strategic situation that the players face in a given state of the dynamic system is akin to a normal form game. Consequently, a Markov perfect equilibrium of a dynamic stochastic game must satisfy the conditions for Nash equilibrium of a certain family of reduced one-shot games. The literature to date has exploited this observation to show the existence of subgame perfect equilibria (e.g., Mertens and Parthasarathy 1987, 1991) and Markov perfect equilibria (e.g., Nowak and Raghavan 1992, Horst 2005) in dynamic stochastic games. Our main insight is that the connection between dynamic stochastic games and normal form games can be used to establish genericity, stability, and purifiability results. Viewing a dynamic stochastic game as a family of interrelated (and endogenous) normal form games, we derive a system of equations, $f(\sigma)=0$, that must be satisfied by any Markov perfect equilibrium $\sigma$. We say that the equilibrium is regular if the Jacobian of $f$ with respect to $\sigma, \partial f(\sigma) / \partial \sigma$, has full rank. Our notion of regularity is closely related to that introduced by Harsanyi (1973b, 1973a) for normal form games and, indeed, reduces to it if players fully discount the future. We are thus able to "import" many of the techniques that have been used to prove these results in the context of normal form games.

The presence of nontrivial dynamics introduces nonlinearities that preclude us from directly applying Harsanyi's (1973a) construction to prove our main genericity result. Two insights are the key to our proof. First, the map that relates a dynamic stochastic game to the payoffs of the family of induced normal form games underlying our regularity notion is linear and invertible. These properties are evident if players fully discount the future but are less than obvious in the presence of nontrivial dynamics. Second, in a departure from the standard treatment in the literature on normal form games 
(Harsanyi 1973a, van Damme 1991), we study the regularity of $f$ by directly applying the transversality theorem-a generalization of Sard's theorem-to it.

As a corollary to our main genericity result, we deduce that almost all dynamic stochastic games have a finite number of Markov perfect equilibria that are locally isolated. This result has already been established in an important paper by Haller and Lagunoff (2000). In contrast to our approach, Haller and Lagunoff (2000) exploit a notion of regularity based on the idea that once-and-for-all deviations from the prescribed equilibrium strategies cannot be profitable. While there are clearly many types of deviations one can consider in dynamic stochastic games, our focus on one-shot deviations has the advantage that it permits us to generalize several other major results for normal form games besides generic finiteness to dynamic stochastic games.

We first show that regular Markov perfect equilibria are robust to slight changes in payoffs and, more specifically, that the equilibria of a given game can be approximated by the equilibria of nearby dynamic stochastic games. To this end, we generalize two stability properties that have received considerable attention in the literature on normal form games to our dynamic setting, namely essentiality and strong stability. Loosely speaking, a Markov perfect equilibrium is essential if it can be approximated by equilibria of nearby games; it is strongly stable if it changes uniquely and continuously with slight changes in payoffs. We show that regular equilibria are strongly stable and, therefore, essential. This result in combination with our main genericity result yields the generic essentiality and strong stability of Markov perfect equilibria. ${ }^{1}$ We, moreover, show that the map from payoffs to equilibria is locally not only continuous, but also differentiable.

These stability properties ensure that slight changes in the parameters of a dynamic stochastic game do not severely alter the nature of the interactions among players. This is especially beneficial if the researcher uses modern econometric techniques (Aguirregabiria and Mira 2007, Bajari et al. 2007, Judd and Su 2008, Pakes et al. 2007, Pesendorfer and Schmidt-Dengler 2008) to estimate the primitives of the game and therefore recovers them with error. In addition, our main stability result lays the foundations for comparative statics. Because the map from payoffs to equilibria is differentiable, differentiable comparative statics are well defined, at least for a small change to the system. We finally discuss how these comparative statics can be numerically implemented using so-called homotopy or path-following methods.

Next we show that regular Markov perfect equilibria admit purification, thereby extending Harsanyi's (1973b) celebrated purification theorem from normal form games to dynamic stochastic games. We perturb a dynamic stochastic game by assuming that, at each decision node, a player's payoffs are subject to random fluctuations that are known to the player but not to his rivals. We demonstrate that any regular Markov perfect equilibrium of the original complete information game can be obtained as the limit of equilibria of the perturbed game of incomplete information as payoff fluctuations become vanishingly small. Hence, one can view the original game of complete information as

\footnotetext{
${ }^{1}$ Maskin and Tirole (2001) demonstrate the generic essentiality of Markov perfect equilibria in finitehorizon dynamic stochastic games. Their result does not imply, nor is it implied by, our essentiality result. Kalandrakis (2006) studies the stability of equilibria in a class of bargaining models.
} 
an idealization of nearby games with a small amount of payoff uncertainty. The proof of our main purification result generalizes arguments previously presented by Govindan et al. (2003) in the context of normal form games. That we are able to do so once again shows the power of our regularity notion.

Our main purification result shows that the choice between complete and incomplete information in formulating a dynamic economic problem is one of convenience, at least when the random payoff fluctuations are small. This is useful because dynamic stochastic games of incomplete information are often easier to solve numerically than their complete information counterparts (Doraszelski and Satterthwaite 2010); they also tend to be more tractable econometrically. Our main purification result further provides a convincing interpretation of behavior strategy equilibria in dynamic stochastic games. Because, at each decision node, a player is indifferent among several pure actions, it is natural to ask what compels the player to randomize precisely as mandated by the equilibrium. In the approximating equilibrium, in contrast, a player is no longer indifferent among several pure actions, but instead has a strictly optimal pure action for almost all realizations of his payoffs.

To the best of our knowledge, our paper is the first to establish the purifiability of equilibrium behavior in a general class of dynamic economic models. The literature so far has studied but a small number of particular examples. Bhaskar (1998) and Bhaskar et al. $(2007,2010)$ provide examples of nonpurifiable equilibria in which strategies depend on payoff irrelevant variables. In contrast, our main purification result shows that equilibria in which strategies depend only on the payoff relevant history are generically purifiable.

In sum, in this paper we develop a theory of regular Markov perfect equilibria in dynamic stochastic games. We show that almost all dynamic stochastic games have a finite number of locally isolated equilibria. These equilibria are essential and strongly stable. Moreover, they all admit purification. The key to obtaining these results is our notion of regularity, which is based on the insight that, holding fixed the value of continued play, the strategic situation that the players face in a given state of the dynamic system is akin to a normal form game. By viewing a dynamic stochastic game as a family of induced normal form games, we are able to make a rich body of literature on normal form games useful for the analysis of dynamic environments.

The remainder of this paper is organized as follows. Section 2 sets out the model and equilibrium concept. Section 3 introduces our notion of regularity and illustrates it with some examples. Section 4 states the main genericity result and discusses its implications for the finiteness of the equilibrium set. Section 5 presents stability properties and Section 6 presents our main purification result. Section 7 contains the proofs of our main genericity and purification results. Supporting arguments have been relegated to the Appendix.

\section{MODEL}

In this section we set up the model and define our notion of equilibrium. We further describe the total payoff that a player receives in a dynamic stochastic game. 


\subsection{Dynamic stochastic games}

A dynamic stochastic game is a dynamic system that can be in different states at different times. Players can influence the evolution of the state through their actions. The goal of a player is to maximize the expected net present value of his stream of payoffs.

We study dynamic stochastic games with finite sets of players, states, and actions. ${ }^{2}$ Let $I$ denote the set of players, let $S$ denote the set of states, and let $A_{i}(s)$ denote the set of actions of player $i$ at state $s$. Time is discrete and the horizon is infinite. ${ }^{3}$

The game proceeds as follows. The dynamic system starts at time $t=0$ from an initial state $s^{t=0}$ that is randomly drawn according to the probability distribution $\bar{q}(\cdot) \in \Delta(S)$, where $\Delta(S)$ denotes the space of probability distributions over $S$. After observing the initial state, players choose their actions $a^{t=0}=\left(a_{i}^{t=0}\right)_{i \in I} \in \prod_{i \in I} A_{i}\left(s^{t=0}\right)=A\left(s^{t=0}\right)$ simultaneously and independently from each other. Now two things happen, depending on the state $s^{t=0}$ and the actions $a^{t=0}$. First, player $i$ receives a payoff $u_{i}\left(a^{t=0}, s^{t=0}\right) \in \mathbb{R}$, where $u_{i}(\cdot, s): A(s) \rightarrow \mathbb{R}$ is the period payoff function of player $i$ at state $s \in S$. Second, the dynamic system transits from state $s^{t=0}$ to state $s^{t=1}$ according to the probability distribution $q\left(\cdot ; a^{t=0}, s^{t=0}\right) \in \Delta(S)$, with $q\left(s^{t=1} ; a^{t=0}, s^{t=0}\right)$ being the probability that state $s^{t=1}$ is selected. In the next round at time $t=1$, after observing the current state $s^{t=1}$, players choose their actions $a^{t=1} \in A\left(s^{t=1}\right)$. Then players receive period payoffs $u\left(a^{t=1}, s^{t=1}\right)$ and the state of the dynamic system changes again. The game goes on in this way ad infinitum.

We let $U_{i}=\left(u_{i}(a, s)\right)_{a \in A(s), s \in S} \in \mathbb{R}^{\sum_{s \in S}|A(s)|}$ denote the vector of payoffs of player $i$ and let $U=\left(U_{i}\right)_{i \in I} \in \mathbb{R}^{|I| \sum_{s \in S}|A(s)|}$ denote the vector of payoffs of all players. A dynamic stochastic game is a tuple

$$
\left\langle S,\left(A_{i}(s)\right)_{i \in I, s \in S}, U,\left(\delta_{i}\right)_{i \in I}, q, \bar{q}\right\rangle,
$$

where $\delta_{i} \in[0,1$ [ is the discount factor of player $i$ that is used to compute his total payoff as the expected net present value of his period payoffs. In the remainder of this paper, unless otherwise stated, we identify a dynamic stochastic game with its period payoffs $U=\left(U_{i}\right)_{i \in I}$.

\subsection{Markov perfect equilibria}

A Markov perfect equilibrium is a subgame perfect equilibrium in which the strategies depend only on the payoff relevant history. Below we provide a formal definition of our equilibrium concept and an alternative characterization that is key to the subsequent analysis.

A stationary Markov behavior strategy (or strategy, for short) for player $i$ is a collection of probability distributions $\left(\sigma_{i}(\cdot, s)\right)_{s \in S}$ such that $\sigma_{i}(\cdot, s) \in \Delta\left(A_{i}(s)\right)$ and $\sigma_{i}\left(a_{i}, s\right)$ is

\footnotetext{
${ }^{2}$ Several papers study equilibrium existence in dynamic stochastic games with a continuum of states and actions (e.g., Mertens and Parthasarathy 1987, Nowak and Raghavan 1992, Horst 2005). While clearly not appropriate for all applications, finite settings like ours have been widely used (see Doraszelski and Pakes 2007 for a survey).

${ }^{3}$ Our results also apply to finite-horizon dynamic stochastic games.
} 
the probability that player $i$ selects action $a_{i} \in A_{i}(s)$ in state $s$. We denote the set of strategies for player $i$ as $\Sigma_{i}=\prod_{s \in S} \Delta\left(A_{i}(s)\right)$ and define $\Sigma=\prod_{i \in I} \Sigma_{i}$. We further extend $u_{i}(\cdot, s)$ and $q\left(s^{\prime} ; \cdot, s\right)$ in the obvious way to allow for randomization over $A(s)$.

Definition 1. A stationary Markov behavior strategy profile $\sigma=\left(\sigma_{i}\right)_{i \in I}$ is a Markov perfect equilibrium (or equilibrium, for short) if it is a subgame perfect equilibrium.

We denote the set of Markov perfect equilibria of the dynamic stochastic game $U$ by $\operatorname{Equil}(U)$. The nonemptiness of $\operatorname{Equil}(U)$ has long been established in the literature (e.g., Fink 1964).

We further provide an alternative characterization of equilibrium that exploits the recursive structure of the model. A strategy profile $\sigma=\left(\sigma_{i}\right)_{i \in I}$ is a Markov perfect equilibrium if and only if (i) for all $i \in I$, there exists a function $V_{i}: S \rightarrow \mathbb{R}$ such that for all $s \in S$,

$$
V_{i}(s)=\max _{a_{i} \in A_{i}(s)} u_{i}\left(\left(a_{i}, \sigma_{-i}(\cdot, s)\right), s\right)+\delta_{i} \sum_{s^{\prime} \in S} V_{i}\left(s^{\prime}\right) q\left(s^{\prime} ;\left(a_{i}, \sigma_{-i}(\cdot, s)\right), s\right)
$$

and (ii) for all $s \in S$, the strategy profile $\sigma(\cdot, s)=\left(\sigma_{i}(\cdot, s)\right)_{i \in I}$ is a (mixed-strategy) Nash equilibrium of the normal form game in which player $i$ chooses an action $a_{i} \in A_{i}(s)$ and, given the action profile $a=\left(a_{i}\right)_{i \in I} \in A(s)$, obtains a payoff

$$
u_{i}(a, s)+\delta_{i} \sum_{s^{\prime} \in S} V_{i}\left(s^{\prime}\right) q\left(s^{\prime} ; a, s\right) .
$$

The function $V_{i}: S \rightarrow \mathbb{R}$ in equation (2.1) is the equilibrium value function for player $i$. The number $V_{i}(s)$ is the expected net present value of the stream of payoffs to player $i$ if the dynamic system is currently in state $s$. That is, $V_{i}(s)$ is the equilibrium value of continued play to player $i$ starting from state $s$.

Our alternative characterization of equilibrium is based on the observation that, given continuation values, the strategic situation that the players face in a given state $s$ is akin to a normal form game. Consequently, an equilibrium of the dynamic stochastic must induce a Nash equilibrium in a certain reduced one-shot game. The payoff to player $i$ in this game as given in equation (2.2) is the sum of his period payoff and his appropriately discounted continuation value. Note that, given continuation values, equation (2.2) can be used to construct the entire payoff matrix of the normal form game that players face in state $s$.

While simple, the observation that a dynamic stochastic game can be studied by analyzing a family of normal form games is the key to the subsequent analysis. It suggests defining a notion of regularity with reference to the induced normal form games. We formalize this idea in Section 3. The obvious difficulty that we have to confront is that the induced normal form games are endogenous in that the payoffs depend on the equilibrium of the dynamic stochastic game.

\subsection{Notation and continuation values}

Before defining our notion of regularity, we introduce some notation and further describe the total payoff that a player receives in a dynamic stochastic game. 
In what follows, we consider not only equilibria, but also deviations from equilibrium strategies. We thus have to know the value of continued play given an arbitrary strategy profile $\sigma \in \Sigma$. In fact, since $\Sigma$ is not an open set of $\mathbb{R}^{\sum_{i \in I} \sum_{s \in S}\left|A_{i}(s)\right|}$, we work mostly with the set $\Sigma^{\epsilon}$. We construct $\Sigma^{\epsilon}$ to be open in $\mathbb{R}^{\sum_{i \in I} \sum_{s \in S}\left|A_{i}(s)\right|}$ and to strictly contain $\Sigma$. The construction of $\Sigma^{\epsilon}$ is detailed in the Appendix. Here we just note that $\Sigma^{\epsilon}$ has elements that are not strategies. Throughout the paper, we reserve the term "strategy profile" for an element of $\Sigma$.

To facilitate the subsequent analysis, we introduce some notation. Enumerate the action profiles available at state $s$ as

$$
A(s)=\left\{a_{s}^{1}, \ldots, a_{s}^{|A(s)|}\right\} .
$$

We also write $S=\left\{s_{1}, \ldots, s_{|S|}\right\}$. As in Haller and Lagunoff (2000), we define the transition matrix $Q \in \mathbb{R}^{\sum_{s \in S}|A(s)| \times|S|}$ as

$$
\left(\begin{array}{ccc}
q\left(s_{1} ; a_{s_{1}}^{1}, s_{1}\right) & \cdots & q\left(s_{|S|} ; a_{s_{1}}^{1}, s_{1}\right) \\
q\left(s_{1} ; a_{s_{1}}^{2}, s_{1}\right) & \cdots & q\left(s_{|S|} ; a_{s_{1}}^{2}, s_{1}\right) \\
\vdots & & \vdots \\
q\left(s_{1} ; a_{s_{1}}^{\left|A\left(s_{1}\right)\right|}, s_{1}\right) & \cdots & q\left(s_{|S|} ; a_{s_{1}}^{\left|A\left(s_{1}\right)\right|}, s_{1}\right) \\
q\left(s_{1} ; a_{s_{2}}^{1}, s_{2}\right) & \cdots & q\left(s_{|S|} ; a_{s_{2}}^{1}, s_{2}\right) \\
\vdots & & \vdots \\
q\left(s_{1} ; a_{s_{2}}^{\left|A\left(s_{2}\right)\right|}, s_{2}\right) & \cdots & q\left(s_{|S|} ; a_{s_{2}}^{\left|A\left(s_{2}\right)\right|}, s_{2}\right) \\
\vdots & & \vdots \\
q\left(s_{1} ; a_{s_{|S|} \mid}^{1}, s_{|S|}\right) & \cdots & q\left(s_{|S|} ; a_{s_{|S|} \mid}^{1}, s_{|S|}\right) \\
\vdots & & \vdots \\
q\left(s_{1} ; a_{s_{|S|}}^{\left|A\left(s_{|S|}\right)\right|}, s_{|S|}\right) & \cdots & q\left(s_{|S|} ; a_{s_{|S|} \mid}^{\left|A\left(s_{|S|}\right)\right|}, s_{|S|}\right)
\end{array}\right) .
$$

We further define the matrix $\mathcal{P}_{\sigma} \in \mathbb{R}^{|S| \times \sum_{s \in S}|A(s)|}$ as

$$
\left(\begin{array}{cccccccccc}
\sigma\left(a_{s_{1}}^{1}, s_{1}\right) & \cdots & \sigma\left(a_{s_{1}}^{\left|A\left(s_{1}\right)\right|}, s_{1}\right) & 0 & \cdots & 0 & \cdots & 0 & \cdots & 0 \\
0 & \cdots & 0 & \sigma\left(a_{s_{2}}^{1}, s_{2}\right) & \cdots & \sigma\left(a_{s_{2}}^{\left|A\left(s_{2}\right)\right|}, s_{2}\right) & \cdots & 0 & \cdots & 0 \\
\vdots & & \vdots & \vdots & & \vdots & & \vdots & & \vdots \\
0 & \cdots & 0 & 0 & \cdots & 0 & \cdots & \sigma\left(a_{s|S|}^{1}, s_{|S|}\right) & \cdots & \sigma\left(a_{S S \mid}^{|A| S|S|) \mid}, s_{|S|}\right)
\end{array}\right) .
$$

In the remainder of the paper, we write $\mathcal{P}_{\sigma}^{s}$ to denote row $s$ of $\mathcal{P}_{\sigma}$. We also define $\mathcal{P}_{a_{i}, \sigma_{-i}}^{s}$ as the $s$ row of the matrix above, assuming that player $i$ chooses $a_{i} \in A_{i}(s)$ with probability 1 in state $s$. Finally, $\mathbb{I}_{r}$ denotes the $\mathbb{R}^{r \times r}$ identity matrix.

Using this notation, the value of continued play given an arbitrary profile $\sigma \in \Sigma^{\epsilon}$ is

$$
V_{i}^{U}(\cdot, \sigma)=\left(\sum_{t=0}^{\infty}\left(\delta_{i}\right)^{t}\left(\mathcal{P}_{\sigma} Q\right)^{t} \mathcal{P}_{\sigma}\right) U_{i}=\left(\mathbb{I}_{|S|}-\delta_{i} \mathcal{P}_{\sigma} Q\right)^{-1} \mathcal{P}_{\sigma} U_{i},
$$

where the inversion is justified by the construction of $\Sigma^{\epsilon}$. We interpret $V_{i}^{U}(s, \sigma)$ as the expected net present value of the stream of payoffs to player $i$ if the dynamic system 
is currently in state $s$ and play is according to $\sigma$. Our notation emphasizes that these continuation values also depend on the payoff vector $U$.

\section{Regularity}

In this section we first define our notion of regularity. Then we illustrate our definition with two examples.

\subsection{Regular Markov perfect equilibria}

Our notion of regularity is based on the observation that, given continuation values, the strategic situation that the players face in a given state $s$ is akin to a normal form game with the payoffs in equation (2.2). Since an equilibrium of the dynamic stochastic game must induce a Nash equilibrium of this normal form game, one-shot deviations cannot be profitable.

To make this idea precise, for all $s \in S$, define $\mathcal{U}_{i}(\cdot, s, \cdot): A_{i}(s) \times \Sigma^{\epsilon} \rightarrow \mathbb{R}$ by

$$
\mathcal{U}_{i}\left(a_{i}, s, \sigma\right)=u_{i}\left(\left(a_{i}, \sigma_{-i}(\cdot, s)\right), s\right)+\delta_{i} \sum_{s^{\prime} \in S} V_{i}^{U}\left(s^{\prime}, \sigma\right) q\left(s^{\prime} ;\left(a_{i}, \sigma_{-i}(\cdot, s)\right), s\right) .
$$

The number $\mathcal{U}_{i}\left(a_{i}, \sigma, s\right)$ is the expected net present value of the stream of payoffs to player $i$ if the current state is $s$, his rivals play according to $\sigma_{-i}$, and player $i$ chooses action $a_{i}$ in the current period and then plays according to $\sigma_{i}$ from the subsequent period on. If $\sigma \in \Sigma$ is an equilibrium, then one-shot deviations cannot be profitable. Formally, if $\sigma \in \Sigma$ is an equilibrium, then, for all $i \in I$ and $s \in S$,

$$
\sigma_{i}\left(a_{i}, s\right)>0 \quad \text { implies } a_{i} \in \arg \max _{a_{i}^{\prime} \in A_{i}(s)} \mathcal{U}_{i}\left(a_{i}^{\prime}, s, \sigma\right) .
$$

These are the conditions for a Nash equilibrium in the normal form game in state $s$ as induced by the value of continued play $V_{i}^{U}(\cdot, \sigma)$.

Consider a collection of actions $a_{i}^{s} \in A_{i}(s)$ for all $i \in I$ and $s \in S$. We think of $a_{i}^{s}$ as a reference action for player $i$ in state $s$. We now define $f: \Sigma^{\epsilon} \times \mathbb{R}^{|I| \sum_{s \in S}|A(s)|} \rightarrow$ $\mathbb{R}^{\sum_{i \in I} \sum_{s \in S}\left|A_{i}(s)\right|}$ so that its $\left(i, a_{i}, s\right)$ component is given by

$$
f_{i, a_{i}, s}(\sigma, U)= \begin{cases}\sum_{a_{i} \in A_{i}} \sigma_{i}\left(a_{i}, s\right)-1 & \text { if } a_{i}=a_{i}^{s} \\ \sigma_{i}\left(a_{i}, s\right)\left(\mathcal{U}_{i}\left(a_{i}, s, \sigma\right)-\mathcal{U}_{i}\left(a_{i}^{s}, s, \sigma\right)\right) & \text { if } a_{i} \in A_{i}(s) \backslash\left\{a_{i}^{s}\right\} .\end{cases}
$$

Condition (3.2) implies that if $\sigma$ is an equilibrium of the game $U$ such that $\sigma\left(a_{i}^{s}, s\right)>0$ for all $i \in I$ and $s \in S$, then

$$
f(\sigma, U)=0 .
$$

Equation (3.4) is necessary but not sufficient for an equilibrium. Further, since $\mathcal{P}_{\sigma}$ is continuously differentiable as a function of $\sigma \in \Sigma^{\epsilon}$, so are $V_{i}^{U}(s, \sigma)$ and $f(\sigma, U)$ as functions of $\sigma \in \Sigma^{\epsilon}$. For future reference, we note that $f$ is also continuously differentiable as a function of $(\sigma, U) \in \Sigma^{\epsilon} \times \mathbb{R}^{|I| \sum_{s \in S}|A(s)|}$.

We are now ready to define our notion of regularity. 
Definition 2. A Markov perfect equilibrium $\sigma$ of a dynamic stochastic game $U$ is regular if the Jacobian of $f$ with respect to $\sigma, \partial f(\sigma, U) / \partial \sigma$, has full rank for some selection of actions $a_{i}^{s} \in A_{i}$ such that $\sigma\left(a_{i}^{s}, s\right)>0$ for all $i \in I$ and $s \in S$. An equilibrium is irregular if it is not regular.

The definition of $f$ depends on the reference actions. However, if an equilibrium $\sigma$ is regular given a collection $\left(a_{i}^{s}\right)_{i \in I, s \in S}$ with $\sigma_{i}\left(a_{i}^{s}, s\right)>0$, then it is also regular given another collection $\left(b_{i}^{s}\right)_{i \in I, s \in S}$ with $\sigma_{i}\left(b_{i}^{s}, s\right)>0$. The same invariance holds true in normal form games (van Damme 1991, Lemma 2.5.4); it arises because the Jacobian of $f$ when using $\left(a_{i}^{s}\right)_{i \in I, s \in S}$ as reference actions is, up to elementary algebraic operations, the same as the Jacobian of $f$ when using $\left(b_{i}^{s}\right)_{i \in I, s \in S}$.

Our definition of regularity is reminiscent of that introduced by Harsanyi (1973a, $1973 \mathrm{~b}$ ) for normal form games. Indeed, if $\delta_{i}=0$ for all $i \in I$, our notion of regularity reduces to the standard notion. But even if $\delta_{i}>0$ for some $i \in I$, our notion remains closely related to the standard notion because we base it on the equilibrium conditions for reduced one-shot games. This permits us to make a rich body of literature on normal form games useful for the analysis of dynamic environments.

\subsection{Examples}

We provide two examples of dynamic stochastic games having regular equilibria. While our main genericity result (Theorem 1) shows that regularity is a property that is satisfied by all equilibria of a large set of models, any particular application may not be part of this set. Regularity may therefore have to be established from first principles. Our examples show that doing so is relatively straightforward. Because the equilibria in our examples are regular, they are robust to slight changes in payoffs (Proposition 2) and the introduction of incomplete information (Theorem 2).

EXAMPLE 1 (Exit game). We consider a simple version of the model of industry dynamics proposed by Ericson and Pakes (1995) (see also Doraszelski and Satterthwaite 2010). Consider an industry with two firms. The set of players is $I=\{1,2\}$ and the set of states is $S=\{(1,1),(1,0),(0,1),(0,0)\}$, where the state $s=\left(s_{1}, s_{2}\right)$ indicates whether firm $i$ is in the market $\left(s_{i}=1\right)$ or out of the market $\left(s_{i}=0\right)$.

We assume that the only nontrivial decision a firm has to make is whether or not to exit if the market is a duopoly; in all other states, a firm has no choice but to preserve the status quo. More formally, firm $i$ 's action set is $\{$ exit, stay\} in state $s=(1,1)$ and a singleton in all other states. All states other than state $(1,1)$ are absorbing.

If the market is a duopoly in state $(1,1)$, then each firm receives a period payoff $\pi^{D}$. If the market is a monopoly in state $(1,0)$ or $(0,1)$, then the monopolist receives a period payoff $\pi^{M}$ and its competitor receives nothing. Neither firm receives anything in state $(0,0)$. Finally, if a firm is in the market but decides to exit it, then it receives a scrap value $\phi$, regardless of what the other firm does. We assume that

$$
\frac{\delta}{1-\delta} \pi^{D}<\phi<\frac{\delta}{1-\delta} \pi^{M}
$$

where $\delta \in] 0,1$ [ is the common discount factor. Hence, while a monopoly is viable, a duopoly is not. The duopolists are thus caught up in a war of attrition. 
The exit game has three equilibria, two of them in pure strategies. In the purestrategy equilibria, one of the firms exits in state $(1,1)$ while the other stays. At each decision node, a firm strictly prefers to conform with the equilibrium strategy (we call these equilibria strict; see Section 5.1 for a formal definition). Proposition 1 in Section 5.1 therefore implies that these equilibria are regular.

The symmetric equilibrium, denoted by $\bar{\sigma}$, is fully characterized by the probability of exiting if the market is a duopoly:

$$
\bar{\sigma}_{i}(\text { exit, }(1,1))=\frac{(1-\delta) \phi-\delta \pi^{D}}{\delta\left(\frac{\pi^{M}}{1-\delta}-\pi^{D}-\phi\right)} .
$$

Our goal here is to show that this mixed-strategy equilibrium is regular.

To compute the value of continued play given an arbitrary strategy profile $\sigma$, we exploit the simple structure of the exit game rather than rely on equation (2.3). Since all states other than state $(1,1)$ are absorbing, we have $V_{i}(s, \sigma)=0$ if $s_{i}=0$ and $V_{i}(s, \sigma)=$ $\pi^{M} /(1-\delta)$ if $s_{i}=1$ and $s_{-i}=0$. The value of continued play in state $(1,1), V_{i}((1,1), \sigma)$, is defined recursively as the unique solution to

$$
\begin{aligned}
& V_{i}((1,1), \sigma) \\
& \qquad \pi^{D}+\sigma_{i}(\operatorname{exit},(1,1))\{\phi+\delta 0\} \\
& \quad+\sigma_{i}(\text { stay, }(1,1))\left\{\delta \sigma_{-i}(\operatorname{exit},(1,1)) \frac{\pi^{M}}{1-\delta}+\delta \sigma_{-i}(\text { stay, }(1,1)) V_{i}((1,1), \sigma)\right\} .
\end{aligned}
$$

The $\left(i, a_{i}, s\right)$ component of $f$ is

$f_{i, a_{i}, s}(\sigma)= \begin{cases}\sigma_{i}\left(a_{i}, s\right)-1 & \text { if } s_{i} \neq(1,1) \\ \sigma_{i}(\text { exit },(1,1))+\sigma_{i}(\text { stay },(1,1))-1 & \text { if } s_{i}=(1,1) \text { and } a_{i}=\text { stay } \\ \sigma_{i}(\operatorname{exit},(1,1))\left\{\pi^{D}+\phi-\left(\pi^{D}+\delta \sigma_{-i}(\text { exit, }(1,1)) \frac{\pi^{M}}{1-\delta}\right.\right. \\ \left.\left.+\delta \sigma_{-i}(\text { stay, }(1,1)) V_{i}((1,1), \sigma)\right)\right\} & \text { if } s_{i}=(1,1) \text { and } a_{i}=\text { exit. }\end{cases}$

Computing the Jacobian of $f$ with respect to $\sigma$ and evaluating its determinant at $\bar{\sigma}$, it can be verified that the determinant is nonzero under our assumptions on the parameters, so that the mixed-strategy equilibrium $\bar{\sigma}$ is regular. This is easiest to see if we normalize $\pi^{D}=0$ to reduce the determinant to

$$
-\frac{\delta^{2} \phi^{2}\left(\pi^{M}-(1-\delta) \phi\right)^{4}}{\left(\delta\left(\pi^{M}\right)^{2}-(1-\delta)^{2} \phi^{2}\right)^{2}}<0 .
$$

Example 2 (Repeated prisoners' dilemma). We consider a repeated prisoners' dilemma with two players $I=\{1,2\}$, two actions $A_{i}=\{C, D\}$ per player, and a common discount factor $\delta \in] 0,1[$. The payoff matrix is

\begin{tabular}{c|cc|cc|} 
& \multicolumn{2}{c}{$C$} & \multicolumn{2}{c}{$D$} \\
\cline { 2 - 5 }$C$ & 1 & 1 & $-g$ & $1+g$ \\
\cline { 2 - 5 }$D$ & $1+g$ & $-g$ & 0 & 0 \\
\cline { 2 - 5 } & &
\end{tabular}


Monitoring is public and perfect. From here on we assume $g>0$ and $\delta>g /(1+g)$.

As often is done in the literature on repeated games (e.g., Ely and Välimäki 2002, Mailath and Morris 2002, Phelan and Skrzypacz 2009), we focus on finite state strategies. A finite state strategy for player $i$ is a probability distribution over actions $\sigma_{i}(\cdot, s) \in \Delta\left(A_{i}\right)$ for all states $s \in S$. We view a subgame perfect equilibrium of the repeated game as a Markov perfect equilibrium of a dynamic stochastic game. To illustrate the construction and represent trigger strategies as finite state strategies, define the state space by $S=$ \{on, off $\}$ and the transition function by

$$
q(\mathrm{on} ; a, s)= \begin{cases}1 & \text { if } a=(C, C) \text { and } s=\text { on } \\ 0 & \text { otherwise }\end{cases}
$$

and $\bar{q}(\mathrm{on})=1$. Then player $i$ 's trigger strategy can be represented as

$$
a_{i}(s)= \begin{cases}C & \text { if } s=\text { on } \\ D & \text { otherwise }\end{cases}
$$

This strategy profile is a strict equilibrium of the dynamic stochastic game and therefore is regular by Proposition 1 in Section 5.1.

There is another pure-strategy equilibrium that has both players defect in any state. Again this equilibrium is strict and therefore is regular. Finally, there is a symmetric mixed-strategy equilibrium $\bar{\sigma}$ given by

$$
\bar{\sigma}_{i}(D, \text { off })=1, \quad \bar{\sigma}_{i}(C, \text { on })=\sqrt{\frac{g}{\delta(1+g)}} .
$$

To study the regularity of $\bar{\sigma}$, we compute the determinant of the Jacobian of $f$ at $\bar{\sigma}$ as a function of $g$ and $\delta$. This determinant is zero only when

$$
\delta \in\left\{0,1,-4\left(1+2 g+g^{2}\right) g^{4}\right\}
$$

Since $\delta \in[g /(1+g), 1[$, the mixed-strategy equilibrium $\bar{\sigma}$ is regular and thus strongly stable and purifiable. ${ }^{4}$

Purifiability of equilibria in dynamic models has been studied by Bhaskar (1998) and Bhaskar et al. (2007, 2010). Bhaskar et al. (2007) study the purifiability of belief-free equilibria, a class of subgame perfect equilibria in stationary behavior strategies with one-period memory introduced by Ely and Välimäki (2002). They show that in the repeated prisoners' dilemma there is a continuum of belief-free equilibria and that none of them is purifiable. Our example shows that it is possible to have equilibria that involve nontrivial mixing and yet avoid the purification failure found in these previous studies.

\footnotetext{
${ }^{4}$ Embedding a repeated game into a dynamic stochastic game generalizes beyond our repeated prisoners' dilemma. For example, the strategies used in the proof of Theorem 2 in Fudenberg and Maskin (1986) can be represented as finite state strategies and incentives can be taken to be strict, both on and off path. Therefore, these folk theorem strategies are regular.
} 


\section{Genericity of Regular EQUilibria}

Before demonstrating that our notion of regularity is useful for characterizing the equilibrium set and the properties of its members, we show that regularity is a property that is satisfied by all equilibria of a large set of models.

Recall that we identify a dynamic stochastic game with its period payoff functions $\left(u_{i}\right)_{i \in I}$. We endow the set of games with the Lebesgue measure $\lambda$ and say that a property is generic if it does not hold at most on a closed subset of measure zero. In this case, we say that the property holds for almost all games $U \in \mathbb{R}^{|I| \sum_{s \in S}|A(s)|}$.

The following is the main result of this section.

Theorem 1. For almost all games $U \in \mathbb{R}^{|I| \sum_{s \in S}|A(s)|}$, all equilibria are regular.

The proof of Theorem 1 is detailed in Section 7.1. It proceeds as follows. We first consider the set of dynamic stochastic games having equilibria in which some player puts zero weight on some of his best replies. The set of such games has a small dimension and so does, therefore, the subset of games having irregular equilibria. We then consider the set of games having equilibria in which all players put positive weight on all their best replies (we call these equilibria quasistrict; see Section 5.1 for a formal definition). Within this class, we restrict attention to completely mixed equilibria. For these equilibria, we show that the Jacobian of $f$ with respect to the pair $(\sigma, U)$ has full rank. An application of the transversality theorem-a generalization of Sard's theorem - then yields the desired result.

In the context of normal form games, Harsanyi (1973a) proves the generic regularity of Nash equilibria as follows. Denoting the space of normal form games by $\Gamma$, he constructs a subspace $\bar{\Gamma}$ of the space of games and a function $\Phi: \Sigma \times \bar{\Gamma} \rightarrow \Gamma$ such that $\sigma$ is a regular equilibrium of the game $U \in \Gamma$ if and only if $\Phi(\sigma, \bar{U})=U$ and $\partial \Phi(\sigma, \bar{U}) / \partial(\sigma, \bar{U})$ has full rank, where $\bar{U}$ denotes the projection of $U \in \Gamma$ on $\bar{\Gamma}$. Applying Sard's theorem to $\Phi$, it follows that the set of normal form games having equilibria that are all regular has full Lebesgue measure.

The presence of nontrivial dynamics introduces nonlinearities that preclude us from directly applying Harsanyi's (1973a) construction. Since his proof exploits the polynomial nature of $f$ for normal form games to construct the map $\Phi$, it is not clear how his approach can be extended to our problem. Indeed, the family of induced normal form games is endogenous to the equilibrium of the dynamic stochastic game. Moreover, the value of continued play in equation (2.3) is not a polynomial function of $\sigma$.

Two insights facilitate our analysis. The first is that to enable study of the regularity of $f$, we can apply the transversality theorem directly to it (see Section 7.1.2 for details). The second insight that facilitates our analysis is that, given a strategy profile $\sigma$ to be followed from next period on, the map that relates a dynamic stochastic game to the payoff matrices of the family of induced normal form games is linear and invertible. To see this, consider the normal form game induced in state $s$, given continuation play $\sigma$. Given the action profile $a \in A(s)$, player $i$ obtains a payoff

$$
u_{i}(a, s)+\delta_{i} \sum_{s^{\prime} \in S} V_{i}^{U}\left(s^{\prime}, \sigma\right) q\left(s^{\prime} ; a, s\right),
$$


where we have replaced the equilibrium continuation value $V_{i}(\cdot)$ in equation (2.2) with $V_{i}^{U}(\cdot, \sigma)$, the value of continued play given the arbitrary strategy profile $\sigma$ to be followed from next period on. The payoff to player $i$ in equation (4.1) is the $(a, s)$ component of the vector $U_{i}+\delta_{i} Q V_{i}^{U}(\cdot, \sigma) \in \mathbb{R}^{\sum_{s \in S}|A(s)|}$. Using equation (2.3), we obtain

$$
\begin{aligned}
U_{i}+\delta_{i} Q V_{i}^{U}(\cdot, \sigma) & =\left(\mathbb{I}_{\sum_{s \in S}|A(s)|}+\delta_{i} Q \sum_{t=0}^{\infty}\left(\delta_{i}\right)^{t}\left(\mathcal{P}_{\sigma} Q\right)^{t} \mathcal{P}_{\sigma}\right) U_{i} \\
& =\left(\mathbb{I}_{\sum_{s \in S}|A(s)|}+\delta_{i} Q \mathcal{P}_{\sigma}+\delta_{i} Q \delta_{i}\left(\mathcal{P}_{\sigma} Q\right) \mathcal{P}_{\sigma}+\delta_{i} Q \delta_{i}^{2}\left(\mathcal{P}_{\sigma} Q\right)^{2} \mathcal{P}_{\sigma}+\cdots\right) U_{i} \\
& =\left(\sum_{t=0}^{\infty}\left(\delta_{i}\right)^{t}\left(Q \mathcal{P}_{\sigma}\right)^{t}\right) U_{i} \\
& =\left(\mathbb{I}_{\sum_{s \in S}|A(s)|}-\delta_{i} Q \mathcal{P}_{\sigma}\right)^{-1} U_{i},
\end{aligned}
$$

where the inversion is justified since all the relevant matrices have strictly dominant diagonals by construction of $\Sigma^{\epsilon}$. The following lemma summarizes the discussion so far.

Lemma 1 (Invertibility lemma). For all $i \in I$ and $\sigma \in \Sigma^{\epsilon}$, the matrix $\left(\mathbb{I}_{\sum_{s \in S}|A(s)|}-\right.$ $\left.\delta_{i} Q \mathcal{P}_{\sigma}\right)^{-1}$ has full rank $\sum_{s \in S}|A(s)|$ and the map $U_{i} \mapsto U_{i}+\delta_{i} Q V_{i}^{U}(\cdot, \sigma)$ is linear and invertible.

Linearity and invertibility are evident for normal form games where the term $\delta_{i} Q V_{i}^{U}(\cdot, \sigma)$ vanishes. In our dynamic setting, the matrix $\left(\mathbb{I}_{\sum_{s \in S}|A(s)|}-\delta_{i} Q \mathcal{P}_{\sigma}\right)^{-1}$ is a part of the Jacobian of $f$ with respect to $U$. The significance of Lemma 1 is thus that it enables us to determine the rank of the Jacobian of $f$ with respect to the pair $(\sigma, U)$, a key step in applying the transversality theorem (see Section 7.1.1 for details).

To fully appreciate the importance of Lemma 1, fix the continuation play and consider the set of reduced normal form games induced by all period payoffs $U \in$ $\mathbb{R}^{|I| \sum_{s \in S}|A(s)|}$. If $\delta_{i}=0$ for all $i \in I$, then the set of reduced normal form games coincides with the set of all possible normal form games and, therefore, the set of games $U$ having equilibria that are all regular is generic. If $\delta_{i}>0$ for some $i \in I$, then Lemma 1 shows that the set of dynamic stochastic games induces a set of reduced normal form games that has the same dimension as the set of normal form games. Since our regularity notion is in reference to these reduced normal form games, Lemma 1 shows that in the set of all possible such games, we have enough degrees of freedom to prove our genericity result.

To provide a first glimpse at the power of our regularity notion, we note that any regular equilibrium is locally isolated as a consequence of the implicit function theorem. A dynamic stochastic game having equilibria that are all regular has a compact equilibrium set that consists of isolated points; therefore, the equilibrium set has to be finite. We summarize in the following corollary.

Corollary 1 (Haller and Lagunoff 2000). For almost all games $U \in \mathbb{R}^{|I| \sum_{s \in S}|A(s)|}$, the number of equilibria is finite. 
The above result has already been established in an important paper by Haller and Lagunoff (2000). ${ }^{5}$ These authors exploit a notion of regularity derived from the firstorder necessary conditions for an equilibrium of a dynamic stochastic game. This system of equations captures the idea that once-and-for-all deviations from the prescribed equilibrium strategies cannot be profitable. In contrast to Haller and Lagunoff (2000), we focus on deviations from a reduced one-shot game. This approach allows us to derive several economically meaningful properties of regular equilibria. Below we further provide examples of equilibria that are regular in the sense of Haller and Lagunoff (2000) but do not exhibit these properties.

Implicit in the Appendix of Herings and Peeters (2004) is yet another notion of regularity. These authors define a system of equations by exploiting, as we do, the fact that one-shot deviations cannot be profitable. Because in their much larger system of equations the unknowns are the equilibrium strategies, the continuation values, and the Lagrange multipliers associated with nonnegativity constraints (see their Theorem 3.6), their regularity notion is not easily connected to the family of normal form games induced by the value of continued play. While our main stability result can also be proven by deriving additional implications of their implicit regularity notion, it is an open question whether equilibria that are regular in the sense of Herings and Peeters (2004) are also purifiable.

\section{Stability PROPERTIES OF REgUlar EQUilibria}

In this section we explore the notions of strongly stable and of essential equilibria in dynamic stochastic games. Before studying these desirable stability properties, we introduce the notions of strict and quasistrict equilibria. These concepts both help us to clarify the proofs below and are invoked extensively again in Section 7 . At the end of the section, we discuss the implications of our results for applied work.

\subsection{Strict and quasistrict equilibria}

Given a strategy profile $\sigma \in \Sigma$, we define the set of (pure) best replies for player $i$ in state $s$ as

$$
B_{i}(\sigma, s)=\arg \max _{a_{i} \in A_{i}(s)} \mathcal{U}_{i}\left(a_{i}, s, \sigma\right) .
$$

We also define the carrier $C_{i}(\sigma, s) \subseteq A_{i}(s)$ of player $i$ in state $s$ as the set of actions $a_{i}$ with $\sigma_{i}\left(a_{i}, s\right)>0$. We finally define $B(\sigma)=\prod_{i \in I} \prod_{s \in S} B_{i}(\sigma, s)$ and $C(\sigma)=\prod_{i \in I} \prod_{s \in S} C_{i}(\sigma, s)$.

If $\sigma$ is an equilibrium, then $C_{i}(\sigma, s) \subseteq B_{i}(\sigma, s)$ for all $i \in I$ and $s \in S$. The equilibrium is quasistrict if $B_{i}(\sigma, s)=C_{i}(\sigma, s)$ for all $i \in I$ and $s \in S$. This means that all players put strictly positive weight on all their best replies. We further say that the equilibrium is strict if the set of best replies is always a singleton, i.e., $\left|B_{i}(\sigma, s)\right|=1$ for all $i \in I$ and $s \in S$.

\footnotetext{
${ }^{5}$ Herings and Peeters (2004) strengthen Corollary 1 and show that the number of equilibria is generically not only finite but also odd. While not pursued here, it is possible to use our regularity notion to define the index of an equilibrium. This yields, among other properties, the generic oddness of the number of equilibria. Ritzberger (1994) discusses related results for normal form games.
} 
A strict equilibrium is also quasistrict, but how these concepts relate to regularity is not immediately apparent. The following proposition resembles a well known result for normal form games (van Damme 1991, Corollary 2.5.3).

Proposition 1. Every strict equilibrium is regular. Every regular equilibrium is quasistrict.

Proof. Define $J(\sigma)=\partial f(\sigma, U) / \partial \sigma$ to be the Jacobian of $f$ with respect to $\sigma$, and consider the submatrix $\bar{J}(\sigma)$ obtained from $J(\sigma)$ by crossing out all columns and rows corresponding to components $\left(a_{i}, s\right)$ with $a_{i} \in A_{i}(s) \backslash C_{i}(\sigma, s)$. For all pairs $\left(a_{i}, s\right)$ with $a_{i} \notin C_{i}(\sigma, s)$, we have

$$
\frac{\partial f_{i, a_{i}, s}(\sigma, U)}{\partial \sigma_{i}\left(a_{i}, s\right)}=\mathcal{U}_{i}\left(a_{i}, s, \sigma\right)-\mathcal{U}_{i}\left(a_{i}^{s}, s, \sigma\right),
$$

while for all $j \in I$ and $\tilde{a}_{j} \in A_{j}(s)$, with $\tilde{a}_{j} \neq a_{i}$ if $j=i$, we have

$$
\frac{\partial f_{i, a_{i}, s}(\sigma, U)}{\partial \sigma_{j}\left(\widetilde{a}_{j}, s\right)}=0
$$

It follows that

$$
|\operatorname{det}(J(\sigma))|=|\operatorname{det}(\bar{J}(\sigma))|\left|\prod_{i \in I} \prod_{s \in S} \prod_{a_{i} \in A_{i}(s) \backslash C_{i}(\sigma, s)}\left[\mathcal{U}_{i}\left(a_{i}, s, \sigma\right)-\mathcal{U}_{i}\left(a_{i}^{s}, s, \sigma\right)\right]\right| .
$$

If the equilibrium $\sigma \in \Sigma$ is strict, then $\left\{a_{i}^{s}\right\}=C_{i}(\sigma, s)=B_{i}(\sigma, s)$ for all $i \in I$ and $s \in S$. Therefore, $\operatorname{det}(\bar{J}(\sigma))=1$ and $\mathcal{U}_{i}\left(a_{i}, s, \sigma\right)-\mathcal{U}_{i}\left(a_{i}^{s}, s, \sigma\right)<0$ for all pairs $\left(a_{i}, s\right)$ with $a_{i} \notin C_{i}(\sigma, s)$. It follows that $\operatorname{det}(J(\sigma)) \neq 0$ so that $\sigma$ is regular. On the other hand, if the equilibrium is regular, then each of the terms on the right hand side of equation (5.1) is nonzero. Hence, $\mathcal{U}_{i}\left(a_{i}, s, \sigma\right)<\mathcal{U}_{i}\left(a_{i}^{s}, s, \sigma\right)$ for all pairs $\left(a_{i}, s\right)$ with $a_{i} \in A_{i}(s) \backslash C_{i}(\sigma, s)$; this corresponds to the definition of quasistrictness.

\subsection{Strongly stable and essential equilibria}

We now study some continuity properties of regular equilibria with respect the data of the game. Continuity is harder to obtain the more parameters of the game are allowed to vary. In this and the next subsection, we fix the action and state spaces, and identify a dynamic stochastic game $G=(U, \delta, q)$ with the vector of period payoffs, in addition to the collection of discount factors $\left(\delta_{i}\right)_{i \in I}$ and the transition function $q$. In this subsection, we also highlight the dependence of $f$, Equil, and $\mathcal{U}_{i}$ on the game $G$ by writing $f(\sigma, G)$ and $\mathcal{U}_{i}^{G}\left(a_{i}, s, \sigma\right)$.

We say that an equilibrium $\bar{\sigma}$ of game $\bar{G}$ is strongly stable if there exist neighborhoods $\mathcal{N}_{\bar{G}}$ of $\bar{G}$ and $\mathcal{N}_{\bar{\sigma}}$ of $\bar{\sigma}$ such that the map equil: $\mathcal{N}_{\bar{G}} \rightarrow \mathcal{N}_{\bar{\sigma}}$ defined by equil $(G)=$ Equil $(G) \cap \mathcal{N}_{\bar{\sigma}}$ is single-valued and continuous. In words, an equilibrium is strongly stable if the equilibrium correspondence is locally a continuous function. This definition generalizes that introduced for normal form games by Kojima et al. (1985). 
Proposition 2. Every regular equilibrium is strongly stable.

Proof. Let $\bar{\sigma}$ be a regular equilibrium of game $\bar{G}$. Since $\partial f(\bar{\sigma}, \bar{G}) / \partial \sigma$ has full rank, the implicit function theorem implies the existence of open neighborhoods $\mathcal{N}_{\bar{G}}$ of $\bar{G}$ and $\mathcal{N}_{\bar{\sigma}}$ of $\bar{\sigma}$, and of a differentiable function $\widetilde{\sigma}: \mathcal{N}_{\bar{G}} \rightarrow \mathcal{N}_{\bar{\sigma}}$ such that for all $G \in \mathcal{N}_{\bar{G}}$, $\widetilde{\sigma}(G)$ is the unique solution $\sigma \in \mathcal{N}_{\bar{\sigma}}$ to $f(\sigma, G)=0$. We can choose $\mathcal{N}_{\bar{G}}$ and $\mathcal{N}_{\bar{\sigma}}$ small enough so that for all $i \in I$, all $s \in S$, and all $a_{i} \in A_{i}(s)$, the following properties hold: (i) If $\bar{\sigma}_{i}\left(a_{i}, s\right)>0$,

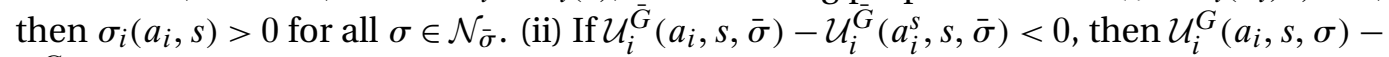
$\mathcal{U}_{i}^{G}\left(a_{i}^{s}, s, \sigma\right)<0$ for all $(\sigma, G) \in \mathcal{N}_{\bar{\sigma}} \times \mathcal{N}_{\bar{G}}$.

Denote $\tilde{\sigma}(G)$ by $\sigma^{G}$. From (i) it follows that $\sigma_{i}^{G}\left(a_{i}, s\right)>0$ for all $a_{i} \in C_{i}(\bar{\sigma}, s)$. This together with the definition of $f$ implies that for all $G \in \mathcal{N}_{\bar{G}}$,

$$
C_{i}(\bar{\sigma}, s) \subseteq C_{i}\left(\sigma^{G}, s\right) \text { and } \mathcal{U}_{i}^{G}\left(a_{i}, s, \sigma^{G}\right)=\mathcal{U}_{i}^{G}\left(a_{i}^{s}, s, \sigma^{G}\right) \text { for } a_{i} \in C_{i}\left(\sigma^{G}, s\right)
$$

Now, for $a_{i} \in A_{i}(s) \backslash C_{i}(\bar{\sigma}, s)$, the fact that $\bar{\sigma}$ is regular and so quasistrict implies that $\mathcal{U}_{i}^{\bar{G}}\left(a_{i}, s, \bar{\sigma}\right)<\mathcal{U}_{i}^{\bar{G}}\left(a_{i}^{s}, s, \bar{\sigma}\right)$. From (ii) it follows that $\mathcal{U}_{i}^{G}\left(a_{i}, s, \sigma^{G}\right)<\mathcal{U}_{i}^{G}\left(a_{i}^{s}, s, \sigma^{G}\right)$ and, by definition of $\sigma^{G}, \sigma^{G}\left(a_{i}, s\right)=0$. It follows that for all $G \in \mathcal{N}_{\bar{G}}$,

$$
C_{i}\left(\sigma^{G}, s\right) \subseteq C_{i}(\bar{\sigma}, s) \text { and } \mathcal{U}_{i}^{G}\left(a_{i}, s, \sigma^{G}\right)<\mathcal{U}_{i}^{G}\left(a_{i}^{s}, s, \sigma^{G}\right) \text { for } a_{i} \notin C_{i}(\bar{\sigma}, s) .
$$

Conditions (5.2) and (5.3) imply that $\sigma^{G} \in \operatorname{Equil}(G)$ for all $G \in \mathcal{N}_{\bar{G}}$. Moreover, $\sigma^{G}$ is the only equilibrium of $G$ in $\mathcal{N}_{\bar{\sigma}}$ because any other equilibrium $\sigma$ would have to satisfy $f(\sigma, G)=0$; consequently, equil $(G)=\operatorname{Equil}(G) \cap \mathcal{N}_{\bar{\sigma}}=\sigma^{G}$. Since $\sigma^{G}$ is differentiable, equil $(G)=\sigma^{G}$ is differentiable (and therefore continuous).

For future reference, we note that the proof of Proposition 2 develops an argument we exploit in the proof of our main purification result (see Section 7.2.2 for details). The idea behind the proof is to show that close enough to a regular equilibrium $\bar{\sigma}$ of $\bar{G}$, the system of equations $f(\sigma, G)=0$ fully characterizes the equilibrium map Equil. This is not self-evident for, as we noted in Section 3.1, $f(\sigma, G)=0$ is necessary but not sufficient for $\sigma \in \operatorname{Equil}(G)$. Hence, to establish the proposition, we further invoke the quasistrictness of a regular equilibrium.

Importantly, the proof of Proposition 2 shows that the equilibrium correspondence is locally not only a continuous, but also a differentiable function.

Corollary 2. Let $\bar{\sigma} \in \operatorname{Equil}(\bar{G})$ be regular. Then there exist open neighborhoods $\mathcal{N}_{\bar{G}}$ of $\bar{G}$ and $\mathcal{N}_{\bar{\sigma}}$ of $\bar{\sigma}$ such that the map equil $: \mathcal{N}_{\bar{G}} \rightarrow \mathcal{N}_{\bar{\sigma}}$ defined by equil $(G)=\operatorname{Equil}(G) \cap \mathcal{N}_{\bar{\sigma}}$ is a differentiable function.

To illustrate, note that the symmetric mixed-strategy equilibrium in Example 1 is regular and therefore differentiable in the parameters of the model.

Turning to the notion of essentiality, we say that an equilibrium $\bar{\sigma}$ of game $\bar{G}$ is essential if, for every neighborhood $\mathcal{N}_{\bar{\sigma}}$, there exists a neighborhood $\mathcal{N}_{\bar{G}}$ such that for all games $G \in \mathcal{N}_{\bar{G}}$ there exists $\sigma \in \operatorname{Equil}(G) \cap \mathcal{N}_{\bar{\sigma}}$. In words, an equilibrium is essential if it can be approximated by equilibria of nearby games. Since any strongly stable equilibrium can be approximated by equilibria of nearby games, the following proposition is immediate. 
Proposition 3. Every strongly stable equilibrium is essential.

Theorem 1, Proposition 2, and Proposition 3 permit us to deduce the generic essentiality of equilibria, generalizing a well known result for normal form games due to $\mathrm{Wu}$ and Jiang (1962) to our dynamic context.

The regularity notion in Haller and Lagunoff (2000) implies neither quasistrictness nor strong stability. Consider an arbitrary two-player game and any equilibrium $\sigma$ satisfying Haller and Lagunoff's (2000) regularity notion. Construct the following artificial game. For some state $s^{\prime}$, add an action $a_{i}^{\prime}$ to the action sets $A_{i}\left(s^{\prime}\right)$ of both players and let $A_{i}^{\prime}(s)$ denote the new action sets. Extend the transition function so that $q\left(s^{\prime} ; a, s^{\prime}\right)=1$ if $a_{i}=a_{i}^{\prime}$ for some $i \in I$. Further, if player 1 plays $a_{1}^{\prime}$ in state $s^{\prime}$ and player 2 plays $a_{2} \neq a_{2}^{\prime}$, then player 1's period payoff equals $\left(1-\delta_{i}\right) \mathcal{U}_{i}\left(a_{i}, s, \sigma\right)$ for some action $a_{i} \in C_{i}(\sigma, s)$; if player 2 plays $a_{2}^{\prime}$, then player 1's period payoff is $u_{1}\left(a^{\prime}, s^{\prime}\right)>\max _{a \in A(s), s \in S} u_{1}(a, s)$. Construct player 2's period payoff so that $u_{2}\left(a_{1}, a_{2}^{\prime}, s^{\prime}\right)$ is sufficiently negative if $a_{1} \neq a_{1}^{\prime}$ while $u_{2}\left(a^{\prime}, s^{\prime}\right)>\max _{a \in A(s), s \in S} u_{2}(a, s)$. The equilibrium $\sigma$ of the original game can be extended to a strategy profile $\sigma^{\prime}$ that puts weight zero on actions $a_{i}^{\prime}$ in the artificial game. Moreover, because their regularity notion does not restrict actions that are not played in equilibrium, the equilibrium $\sigma^{\prime}$ is regular in Haller and Lagunoff's (2000) sense. But, by construction, $\sigma^{\prime}$ is not quasistrict; $\sigma^{\prime}$ is also not strongly stable because a small increase to player 1's period payoff from action $a_{1}^{\prime}$ completely reshapes the equilibrium.

\subsection{Differentiable comparative statics}

Because regular equilibria are strongly stable and, as shown by Corollary 2, the locally defined map equil is a differentiable function, differentiable comparative statics are well defined, at least for small changes to the system. Moreover, these comparative statics can be numerically implemented. Consider a game $\bar{G}$ and a regular equilibrium $\bar{\sigma}$, and suppose we are interested in how the equilibrium changes when we slightly change the data from $\bar{G}$ to $\hat{G}$. We assume, without loss of generality, that $\mathcal{N}_{\bar{G}}$ in Corollary 2 is convex. If $\hat{G}$ is close enough to $\bar{G}$, then $\hat{G}$ belongs to $\mathcal{N}_{\bar{G}}$, the domain of equil. Consider the homotopy function $H: \mathcal{N}_{\bar{\sigma}} \times[0,1] \rightarrow \mathbb{R}^{\sum_{i \in I} \sum_{s \in S}\left|A_{i}(s)\right|}$ defined by

$$
H(\sigma, \tau)=f(\sigma,(1-\tau) \bar{G}+\tau \hat{G}) .
$$

There exists a path $\sigma:[0,1] \rightarrow \mathcal{N}_{\bar{\sigma}}$ that satisfies $H(\sigma(\tau), \tau)=0$ for all $\tau \in[0,1]$. It is parameterized by the homotopy parameter $\tau$ and connects the "old" equilibrium $\sigma(0)=\bar{\sigma}$ at $\bar{G}$ to a "new" equilibrium $\sigma(1)=\hat{\sigma}$ at $\hat{G}$. One way to compute this path is to numerically solve the ordinary differential equation

$$
\frac{d \sigma}{d \tau}(\tau)=-\left[\frac{d f}{d \sigma}(\sigma(\tau),(1-\tau) \bar{G}+\tau \hat{G})\right]^{-1} \frac{\partial f}{\partial G}(\sigma(\tau),(1-\tau) \bar{G}+\tau \hat{G})(\hat{G}-\bar{G})
$$

with initial condition $\sigma(0)=\bar{\sigma}$. See Zangwill and Garcia (1981) for a more detailed discussion of homotopy methods, and Besanko et al. (2010) and Borkovsky et al. (forthcoming) for an application to dynamic stochastic games. 
A particularly vexing problem arises when the game has multiple equilibria. In this case, little is known about which equilibrium is likely be played after a change to the system has occurred. What happens depends on how players adjust to the change. The above discussion suggests singling out $\sigma(1)=\hat{\sigma}$ as the equilibrium that is likely to be played after the original game $\bar{G}$ has been slightly changed. ${ }^{6}$ We leave it to future work to establish formal conditions under which this equilibrium arises from learning dynamics and out-of-equilibrium adjustment processes.

\section{Purification of regular equilibria}

In this section we present our main purification result. We begin by introducing incomplete information into our baseline model of dynamic stochastic games. After presenting our main purification result, we briefly discuss some of its implications for repeated games.

\subsection{Dynamic stochastic games of incomplete information}

Following Harsanyi (1973b), we assume that in every period $t$, after state $s^{t}$ is drawn, player $i$ receives a shock $\eta_{i}^{t} \in \mathbb{R}^{\left|A\left(s^{t}\right)\right|}$ before choosing his action. The shock $\eta_{i}$ is known to player $i$ but not to his rivals. The private shocks are independent across players and periods, and are drawn from a probability distribution $\mu_{i}\left(\cdot ; s^{t}\right)$. We assume that $\mu_{i}(\cdot ; s)$ is differentiable and therefore absolutely continuous with respect to the Lebesgue measure in $\mathbb{R}^{|A(s)|}$. The period payoff of player $i$ is $u_{i}(a, s)+\eta_{i}(a)$, where $\eta_{i}(a)$ denotes the $a$ component of $\eta_{i}$. We extend $\eta_{i}(\cdot)$ in the obvious way to allow for randomization over $A(s)$. We refer to the private information game as the perturbed dynamic stochastic game; it is characterized by a tuple

$$
\left\langle S,\left(A_{i}(s)\right)_{i \in I, s \in S}, U,\left(\delta_{i}\right)_{i \in I},\left(\mu_{i}(\cdot, s)\right)_{i \in I, s \in S}, q, \bar{q}\right\rangle .
$$

A pure strategy for player $i$ is a function $b_{i}\left(s, \eta_{i}\right)$ of the state $s \in S$ and the private shock $\eta_{i} \in \mathbb{R}^{A(s)}$. The equilibrium concept is Bayesian Markov perfect equilibrium. We, however, are not interested in equilibrium strategies, but in equilibrium distributions. A distribution profile $\sigma=\left(\sigma_{i}\right)_{i \in I} \in \Sigma$ is a Markov perfect equilibrium distribution (or equilibrium distribution, for short) if and only if (i) for all $i \in I$, there exists a function $\bar{V}_{i}: S \rightarrow \mathbb{R}$ such that for all $s \in S$,

$$
\begin{aligned}
\bar{V}_{i}(s)=\int\left(\max _{a_{i} \in A_{i}(s)} u_{i}\left(\left(a_{i}, \sigma_{-i}(\cdot, s)\right), s\right)\right. & +\eta_{i}\left(a_{i}, \sigma_{-i}(\cdot, s)\right) \\
& \left.+\delta_{i} \sum_{s^{\prime} \in S} \bar{V}_{i}\left(s^{\prime}\right) q\left(s^{\prime} ;\left(a_{i}, \sigma_{-i}(\cdot, s)\right), s\right)\right) d \mu_{i}\left(\eta_{i} ; s\right),
\end{aligned}
$$

\footnotetext{
${ }^{6}$ Aguirregabiria and Ho (2008) conduct counterfactual experiments in a dynamic stochastic game by assuming the existence of a locally defined differentiable function that relates parameters to equilibria. While their model does not fit exactly into our framework, their analysis illustrates how the existence and differentiability of equil can be exploited in applications.
} 
and (ii) for all $s \in S$, the distribution profile $\sigma(\cdot, s)=\left(\sigma_{i}(\cdot, s)\right)_{i \in I}$ is consistent with a (purestrategy) Bayesian Nash equilibrium of the incomplete information game in which player $i$ chooses an action $a_{i} \in A_{i}(s)$ and, given the action profile $a=\left(a_{i}\right)_{i \in I}$, obtains a payoff

$$
u_{i}(a, s)+\eta_{i}(a)+\delta_{i} \sum_{s^{\prime} \in S} \bar{V}_{i}\left(s^{\prime}\right) q\left(s^{\prime} ; a, s\right),
$$

where by consistent we mean that if $\left(b_{i}\left(s, \eta_{i}\right)\right)_{i \in I}$ is the strategy profile in the Bayesian Nash equilibrium, then $\sigma_{i}\left(a_{i}, s\right)=\int_{\left\{\eta_{i} \mid b_{i}\left(s, \eta_{i}\right)=a_{i}\right\}} d \mu_{i}\left(\eta_{i} ; s\right) .^{7}$

This characterization of Bayesian Markov perfect equilibrium is similar in spirit to the characterization in Section 2.2. The main difference is that here we have a game of incomplete information and therefore the equilibrium concept in the reduced one-shot game is Bayesian Nash equilibrium. Escobar (2008) ensures the existence of a Bayesian Markov perfect equilibrium in the perturbed dynamic stochastic game.

\subsection{Purification: Convergence and approachability}

To explore how good of an approximation to the original (unperturbed) dynamic stochastic game the perturbed game is, we consider, for all $i \in I$ and $s \in S$, a sequence of probability distributions of private shocks $\left(\mu_{i}^{n}(\cdot ; s)\right)_{n \in \mathbb{N}}$ converging to a mass point at $0 \in \mathbb{R}^{|A(s)|}$. We ask whether the corresponding sequence of perturbed games has equilibrium distributions that are getting closer to the equilibria of the original game.

Before answering this question, we provide a precise notion of convergence for a sequence of probability distributions.

Definition 3. The sequence of probability distributions $\left(\mu_{i}^{n}(\cdot ; s)\right)_{n \in \mathbb{N}}$ converges to a mass point at $0 \in \mathbb{R}^{|A(s)|}$ as $n \rightarrow \infty$ if

$$
\lim _{n \rightarrow \infty} \frac{1}{\mu_{i}^{n}\left(R^{n} ; s\right)} \int_{\eta_{i} \in R^{n}}\left\{\max _{a \in A(s)}\left|\eta_{i}(a)\right|\right\} d \mu_{i}^{n}\left(\eta_{i} ; s\right)=0
$$

for any sequence of measurable sets $\left(R^{n}\right)_{n \in \mathbb{N}}$ such that $\mu_{i}^{n}\left(R^{n}\right)>0$.

The perturbations considered by Harsanyi (1973b) satisfy Definition 3. While our approachability result in Theorem 2 remains valid under more general perturbations, we prefer to work with a single convergence notion because both convergence and approachability are desirable properties in applications. Note that Definition 3 is satisfied by, for example, any sequence of probability distributions $\left(\mu_{i}^{n}(\cdot ; s)\right)_{n \in \mathbb{N}}$ such that the support of $\mu_{i}^{n}(\cdot ; s)$ is contained in a ball of radius $r(n)$ centered at $0 \in \mathbb{R}^{|A(s)|}$, where $r(n) \rightarrow 0$ as $n \rightarrow \infty$.

To facilitate the exposition, we define $\operatorname{Equil}^{n}(U)$ to be the set of equilibrium distributions of the perturbed game when players' private shocks are drawn from $\mu^{n}=$ $\left(\mu_{i}^{n}(\cdot ; s)\right)_{i \in I, s \in S}$. The following proposition shows that as the private shocks vanish, any converging sequence of equilibrium distributions for perturbed games converges to an equilibrium of the original game.

\footnotetext{
${ }^{7}$ Given the absolute continuity of $\mu_{i}$, player $i$ has a unique best reply for almost all realizations of $\eta_{i}$.
} 
Proposition 4 (Convergence). Suppose that, for all $i \in I$ and all $s \in S,\left(\mu_{i}^{n}(\cdot ; s)\right)_{n \in \mathbb{N}}$ converges to a mass point at $0 \in \mathbb{R}^{|A(s)|}$ as $n \rightarrow \infty$. Suppose further that $\left(\sigma^{n}\right)_{n \in \mathbb{N}}$, with $\sigma^{n} \in$ Equil $^{n}(U)$, converges to $\bar{\sigma}$ as $n \rightarrow \infty$. Then $\bar{\sigma} \in \operatorname{Equil}(U)$.

The proof of Proposition 4 is detailed in the Appendix. Note that any sequence $\left(\sigma^{n}\right)_{n \in \mathbb{N}} \subseteq \Sigma$ has a converging subsequence and therefore Proposition 4 applies to the subsequence.

The following theorem is the main result of this section. It shows that any regular equilibrium of the original game can be approximated by equilibrium distributions of nearby perturbed games.

Theorem 2 (Approachability). Suppose that, for all $i \in I$ and all $s \in S,\left(\mu_{i}^{n}(\cdot ; s)\right)_{n \in \mathbb{N}}$ converges to a mass point at $0 \in \mathbb{R}^{|A(s)|}$ as $n \rightarrow \infty$. Let $\bar{\sigma}$ be a regular equilibrium of game $U$. Then, for all $\bar{\epsilon}>0$ and all large enough $n$, there exists $\sigma^{n} \in$ Equil $^{n}(U)$ such that $\left\|\sigma^{n}-\bar{\sigma}\right\|<\bar{\epsilon}$.

In conjunction with Theorem 1, Theorem 2 indicates that, for almost all games $U \in \mathbb{R}^{|I| \sum_{s \in S}|A(s)|}$, all equilibria are purifiable. Hence, one can interpret the original game as an idealization of nearby games with a small amount of payoff uncertainty. Our main purification result also blunts a common criticism of the notion of a mixedstrategy equilibrium, namely that a player has no incentive to adhere to the prescribed randomization over his pure actions, since in the approximating equilibrium a player has a strictly optimal pure action for almost all realizations of his payoffs.

It is considerably more difficult to obtain lower hemicontinuity results such as Theorem 2 than closure results such as Proposition 4. The proof of Theorem 2 is detailed in Section 7.2. We first characterize the set of equilibrium distributions of the games of incomplete information as solutions to a fixed point problem. We then use the fixed point characterization and rely on arguments previously presented by Govindan et al. (2003) to derive the existence of an equilibrium distribution close enough to the regular equilibrium $\bar{\sigma}$. That we are able to generalize their proof once again shows the power of our regularity notion. The two key properties satisfied by regular equilibria that we exploit are strong stability and quasistrictness.

An equilibrium that is regular in Haller and Lagunoff's (2000) sense may not be purifiable. In the example discussed at the end of Section 5.2, the introduction of private information distributed symmetrically around zero implies that action $a_{1}^{\prime}$ must be played with strictly positive probability along any sequence of equilibria of the perturbed games that approximates $\sigma^{\prime}$. This implies that the equilibrium $\sigma^{\prime}$ cannot be purified.

\section{Proofs}

In this section, we detail the proofs of our main genericity and purification results in Theorems 1 and 2, respectively. 


\subsection{Proving Theorem 1}

\subsubsection{Two useful lemmata}

We present two lemmata. As a corollary to the second lemma, we further obtain a characterization of the dimension of the equilibrium graph. To facilitate the analysis, we require some notation. Define arbitrary product sets $B^{*}=\prod_{i \in I} \prod_{s \in S} B_{i}^{*}(s)$ and $C^{*}=\prod_{i \in I} \prod_{s \in S} C_{i}^{*}(s)$, where $B_{i}^{*}(s), C_{i}^{*}(s) \in 2^{\left|A_{i}(s)\right|}$. Further define $G\left(B^{*}, C^{*}\right)$ as the set of games having some equilibrium $\sigma$ with best replies $B^{*}$ and carriers $C^{*}$. Formally,

$G\left(B^{*}, C^{*}\right)=\{U \mid$ there exists $\sigma \in \operatorname{Equil}(U)$ such that

$$
\left.B_{i}(\sigma, \cdot)=B_{i}^{*} \text { and } C_{i}(\sigma, \cdot)=C_{i}^{*} \text { for all } i \in I\right\},
$$

where the sets $B_{i}(\sigma, s)$ and $C_{i}(\sigma, s)$ are as defined in Section 5.1. We also define $I\left(B^{*}, C^{*}\right)$ as the set of games that have some irregular equilibrium with best replies $B^{*}$ and carriers $C^{*}$. Clearly, $I\left(B^{*}, C^{*}\right) \subseteq G\left(B^{*}, C^{*}\right)$.

The first lemma shows that the set of games that have some equilibrium that fails to be quasistrict has measure zero. The proof proceeds as follows. We first derive a set of necessary conditions that characterize a game $\bar{U}$ and an equilibrium $\bar{\sigma}$ that fails to be quasistrict. These indifference conditions can be written as a system of equations, $M(\bar{\sigma}, \bar{U})=0$. Since these equations are linearly independent (as shown below in Claim 1), we can derive a locally defined function that maps strategies and some components of the payoff vector $U$ to the entire vector $U$. We then show that the set of all games $G^{\bar{\sigma}, \bar{U}}\left(B^{*}, C^{*}\right)$ that are close to $\bar{U}$ and have some equilibrium that is close to $\bar{\sigma}$ has a small dimension and is therefore negligible (Claim 2). The lemma finally follows by applying this logic to each possible pair $(\bar{\sigma}, \bar{U})$ in a properly chosen way. (Recall from Section 4 that $\lambda$ is the Lebesgue measure on the set of games.)

Lemma 2. If $B^{*} \neq C^{*}$, then $\lambda\left(G\left(B^{*}, C^{*}\right)\right)=0$. It follows that $\lambda\left(I\left(B^{*}, C^{*}\right)\right)=0$.

Proof. Consider a game $\bar{U}$ with an equilibrium $\bar{\sigma}$ such that $B_{i}(\bar{\sigma}, \cdot)=B_{i}^{*}$ and $C_{i}(\bar{\sigma}, \cdot)=$ $C_{i}^{*}$ for all $i \in I$. Because $B^{*} \neq C^{*}$ by assumption, $\bar{\sigma}$ fails to be quasistrict. Fix a collection of actions $a_{i}^{s} \in A_{i}(s)$ such that $a_{i}^{s} \in C_{i}^{*}(s)$ for all $i \in I$ and $s \in S$.

By definition of $B_{i}^{*}(s)$, it must be that $\mathcal{U}_{i}\left(a_{i}, s, \bar{\sigma}\right)=\mathcal{U}_{i}\left(a_{i}^{s}, s, \bar{\sigma}\right)$ for all $a_{i} \in B_{i}^{*}(s)$. In words, player $i$ 's payoff to all his best replies is the same. In matrix notation, $\mathcal{U}_{i}\left(a_{i}, \bar{\sigma}, s\right)$ in equation (3.1) can be written as $\mathcal{P}_{a_{i}, \bar{\sigma}_{-i}}^{s}\left(\mathbb{I}_{\sum_{s \in S}|A(s)|}-\delta_{i} Q \mathcal{P}_{\bar{\sigma}}\right)^{-1} \bar{U}_{i}$, where $\mathcal{P}_{a_{i}, \bar{\sigma}_{-i}}^{s}$ is as defined in Section 2.3. Hence, for all $a_{i} \in B_{i}^{*}(s) \backslash\left\{a_{i}^{s}\right\},\left(\mathcal{P}_{a_{i}, \bar{\sigma}_{-i}}^{s}-\mathcal{P}_{a_{i}^{s}, \bar{\sigma}_{-i}}^{s}\right) \times$ $\left(\mathbb{I}_{\sum_{s \in S}|A(s)|}-\delta_{i} Q \mathcal{P}_{\bar{\sigma}}\right)^{-1} \bar{U}_{i}=0$. For all $a_{i} \in B_{i}^{*}(s) \backslash\left\{a_{i}^{s}\right\}$ and $s \in S$, define the $\left(a_{i}, s\right)$ row of $\overline{\mathcal{P}}_{i, \bar{\sigma}} \in \mathbb{R}^{\sum_{s \in S}\left(\left|B_{i}^{*}(s)\right|-1\right) \times \sum_{s \in S}|A(s)|}$ by $\left(\mathcal{P}_{a_{i}, \bar{\sigma}_{-i}}^{s}-\mathcal{P}_{a_{i}^{s}, \bar{\sigma}_{-i}}^{s}\right)$. Write the indifference conditions for player $i$ as

$$
\overline{\mathcal{P}}_{i, \bar{\sigma}}\left(\mathbb{I}_{\sum_{s \in S}|A(s)|}-\delta_{i} Q \mathcal{P}_{\bar{\sigma}}\right)^{-1} \bar{U}_{i}=0 .
$$

Collect the indifference conditions for all players to obtain the system of equations

$$
M(\bar{\sigma}, \bar{U})=\left(\begin{array}{c}
\overline{\mathcal{P}}_{1, \bar{\sigma}}\left(\mathbb{I}_{\sum_{s \in S}|A(s)|}-\delta_{1} Q \mathcal{P}_{\bar{\sigma}}\right)^{-1} \bar{U}_{1} \\
\vdots \\
\overline{\mathcal{P}}_{|I|, \bar{\sigma}}\left(\mathbb{I}_{\sum_{s \in S}|A(s)|}-\delta_{|I|} Q \mathcal{P}_{\bar{\sigma}}\right)^{-1} \bar{U}_{|I|}
\end{array}\right)=0
$$


Claim 1. The Jacobian $\partial M(\bar{\sigma}, \bar{U}) / \partial U$ has full rank $\sum_{i \in I} \sum_{s \in S}\left(\left|B_{i}^{*}(s)\right|-1\right)$.

Proof. The Jacobian of $M$ with respect to $U$ takes the form

$$
\begin{aligned}
& \frac{\partial M(\bar{\sigma}, \bar{U})}{\partial U} \\
& \quad=\left(\begin{array}{cclc}
\overline{\mathcal{P}}_{1, \bar{\sigma}}\left(\mathbb{I}_{\sum_{s \in S}|A(s)|}-\delta_{1} Q \mathcal{P}_{\bar{\sigma}}\right)^{-1} & 0 & \ldots & 0 \\
0 & \overline{\mathcal{P}}_{2, \bar{\sigma}}\left(\mathbb{I}_{\sum_{s \in S}|A(s)|}-\delta_{2} Q \mathcal{P}_{\bar{\sigma}}\right)^{-1} & \ldots & 0 \\
\vdots & \vdots & & \vdots \\
0 & 0 & \ldots & \overline{\mathcal{P}}_{|I|, \bar{\sigma}}\left(\mathbb{I}_{\sum_{s \in S}|A(s)|}-\delta_{|I|} Q \mathcal{P}_{\bar{\sigma}}\right)^{-1}
\end{array}\right) .
\end{aligned}
$$

The matrix $\overline{\mathcal{P}}_{i, \bar{\sigma}}$ has full rank $\sum_{s \in S}\left(\left|B_{i}^{*}(s)\right|-1\right)$. To see this, note that for all pairs $\left(a_{i}, s\right)$ with $a_{i} \in B_{i}^{*}(s) \backslash\left\{a_{i}^{s}\right\}$, the $\left(a_{i}, s\right)$ row of $\overline{\mathcal{P}}_{i, \bar{\sigma}}$ contains a zero in all those components $\left(a^{\prime}, s^{\prime}\right)$ where either $s^{\prime} \neq s$ or $a_{i}$ is not contained in $a^{\prime}$ (by this we mean that there is no $a_{-i}$ such that $\left.\left(a_{i}, a_{-i}\right)=a^{\prime}\right)$. The $\left(a_{i}, s\right)$ row also contains a nonzero term in some component $\left(a^{\prime}, s\right)$ where $a^{\prime}$ contains $a_{i}$; indeed, $\sum_{a^{\prime} \text { contains } a_{i}} \bar{\sigma}_{-i}\left(a^{\prime} \backslash a_{i}, s\right)=\sum_{a_{-i}} \bar{\sigma}_{-i}\left(a_{-i}, s\right)=1$, where, given $a^{\prime}=\left(a_{i}, a_{-i}\right)$, we take $a^{\prime} \backslash a_{i}=a_{-i}$. This shows that $\overline{\mathcal{P}}_{i, \bar{\sigma}}$ has full rank. The matrix $\left(\mathbb{I}_{\sum_{s \in S}|A(s)|}-\delta_{i} Q \mathcal{P}_{\bar{\sigma}}\right)^{-1}$ has full rank as a consequence of Lemma 1. Taken together, these observations imply that the Jacobian of $M$ with respect to $U$ has full rank $\sum_{i \in I} \sum_{s \in S}\left(\left|B_{i}^{*}(s)\right|-1\right)$.

As a consequence of the implicit function theorem, it is possible to obtain open sets $\mathcal{N}^{1} \subseteq \mathbb{R}^{|I| \sum_{s \in S}|A(s)|-\sum_{i \in I} \sum_{s \in S}\left(\left|B_{i}^{*}(s)\right|-1\right)}, \mathcal{N}^{2} \subseteq \mathbb{R}^{\sum_{i \in I} \sum_{s \in S}\left(\left|B_{i}^{*}(s)\right|-1\right)}$, and $\mathcal{N} \subseteq \Sigma^{\epsilon}$, where $\bar{U} \in$ $\mathcal{N}^{1} \times \mathcal{N}^{2}$ (properly ordered) and $\bar{\sigma} \in \mathcal{N}$, and a function $\Phi: \mathcal{N}^{1} \times \mathcal{N} \rightarrow \mathcal{N}^{2}$ such that for all $\left(\sigma, U^{1}\right) \in \mathcal{N} \times \mathcal{N}^{1}, \Phi\left(U^{1}, \sigma^{1}\right)$ is the unique solution $U^{2} \in \mathcal{N}^{2}$ to $M\left(\sigma,\left(U^{1}, U^{2}\right)\right)=0$. We define the function $H\left(\sigma, U^{1}\right)=\left(U^{1}, \Phi\left(\sigma, U^{1}\right)\right)$. To highlight the dependence of these objects on $\bar{\sigma}$ and $\bar{U}$, we write $\mathcal{N}_{\bar{\sigma}, \bar{U}}^{1}, \mathcal{N}_{\bar{\sigma}, \bar{U}}^{2}, \mathcal{N}_{\bar{\sigma}, \bar{U}}$, and $H_{\bar{\sigma}, \bar{U}}$, respectively. We assume, without loss of generality, that $\mathcal{N}_{\bar{\sigma}, \bar{U}}^{1}, \mathcal{N}_{\bar{\sigma}, \bar{U}}^{2}$, and $\mathcal{N}_{\bar{\sigma}, \bar{U}}$ are balls with rational centers and radii.

Define $G^{\bar{\sigma}, \bar{U}}\left(B^{*}, C^{*}\right)$ as the set of all games that are close enough to $\bar{U}$ and have some equilibrium that is close enough to $\bar{\sigma}$ with the same best replies and carriers as $\bar{\sigma}$. More formally,

$$
\begin{aligned}
G^{\bar{\sigma}, \bar{U}}\left(B^{*}, C^{*}\right)=\left\{U \in \mathcal{N}_{\bar{\sigma}, \bar{U}}^{1} \times \mathcal{N}_{\bar{\sigma}, \bar{U}}^{2} \mid \text { there exists } \sigma \in \operatorname{Equil}(U) \cap \mathcal{N}_{\bar{\sigma}, \bar{U}}\right. \\
\text { with } \left.B_{i}(\sigma, \cdot)=B_{i}^{*} \text { for all } i \in I \text { and } \sigma \in A\left(C^{*}\right)\right\},
\end{aligned}
$$

where $A\left(C^{*}\right)=\left\{\sigma \in \Sigma \mid C_{i}(\sigma, \cdot)=C_{i}^{*}\right.$ for all $\left.i \in I\right\}$. Further define the set

$$
\begin{array}{r}
P^{\bar{\sigma}, \bar{U}}\left(B^{*}, C^{*}\right)=\left\{U \in \mathcal{N}_{\bar{\sigma}, \bar{U}}^{1} \times \mathcal{N}_{\bar{\sigma}, \bar{U}}^{2} \mid \text { there exists }\left(\sigma, U^{1}\right) \in \mathcal{N}_{\bar{\sigma}, \bar{U}}^{1} \times\left(A\left(C^{*}\right) \cap \mathcal{N}_{\bar{\sigma}, \bar{U}}\right)\right. \\
\text { such that } \left.H_{\bar{\sigma}, \bar{U}}\left(\sigma, U^{1}\right)=U\right\}
\end{array}
$$

Clearly, $G^{\bar{\sigma}, \bar{U}}\left(B^{*}, C^{*}\right) \subseteq P^{\bar{\sigma}, \bar{U}}\left(B^{*}, C^{*}\right)$.

Claim 2. $\lambda\left(P^{\bar{\sigma}, \bar{U}}\left(B^{*}, C^{*}\right)\right)=0$. 
Proof. Note that $\operatorname{dim}\left(\mathcal{N}_{\bar{\sigma}, \bar{U}}^{1}\right)=|I| \sum_{s \in S}|A(s)|-\sum_{i \in I} \sum_{s \in S}\left(\left|B_{i}^{*}(s)\right|-1\right) \quad$ and $\operatorname{dim}\left(A\left(C^{*}\right) \cap \mathcal{N}_{\bar{\sigma}, \bar{U}}\right)=\sum_{i \in I} \sum_{s \in S}\left(\left|C_{i}^{*}(s)\right|-1\right)$. Therefore, $\operatorname{dim}\left(\mathcal{N}_{\bar{\sigma}, \bar{U}}^{1} \times\left(A\left(C^{*}\right) \cap \mathcal{N}_{\bar{\sigma}, \bar{U}}\right)\right)=$ $|I| \sum_{s \in S}|A(s)|-\sum_{i \in I} \sum_{s \in S}\left|B_{i}^{*}(s)\right|+\sum_{i \in I} \sum_{s \in S}\left|C_{i}^{*}(s)\right|<|I| \sum_{s \in S}|A(s)|$ for $B_{i}^{*}(s) \neq C_{i}^{*}(s)$ for some $i \in I$ and $s \in S$. Since $P^{\bar{\sigma}, \bar{U}}\left(B^{*}, C^{*}\right)=H_{\bar{\sigma}, \bar{U}}\left(\left(A\left(C^{*}\right) \cap \mathcal{N}_{\bar{\sigma}, \bar{U}}\right) \times \mathcal{N}_{\bar{\sigma}, \bar{U}}^{1}\right)$, the claim follows.

We are now ready to complete the proof of Lemma 2. For each game $\bar{U}$ having some equilibrium $\bar{\sigma}$ such that $B_{i}(\bar{\sigma}, \cdot)=B_{i}^{*}$ and $C_{i}(\bar{\sigma}, \cdot)=C_{i}^{*}$ for all $i \in I$, we can construct the sets $G^{\bar{\sigma}, \bar{U}}\left(B^{*}, C^{*}\right)$ and $P^{\bar{\sigma}, \bar{U}}\left(B^{*}, C^{*}\right)$. Moreover, since the neighborhoods $\mathcal{N}_{\bar{\sigma}, \bar{U}}^{1}, \mathcal{N}_{\bar{\sigma}, \bar{U}}^{2}$, and $\mathcal{N}_{\bar{\sigma}, \bar{U}}$ are chosen from a countable set, it follows that $G\left(B^{*}, C^{*}\right) \subseteq \bigcup_{n \in \mathbb{N}} Q^{n}$, where $Q^{n}=P^{\bar{\sigma}_{n}, \bar{U}_{n}}\left(B^{*}, C^{*}\right)$ is constructed for each of the countable number of neighborhoods. Lemma 2 now follows from Claim 2 by noting that the countable union of measure zero sets has measure zero as well.

The proof of Lemma 2 resembles proofs given for normal form games by Harsanyi (1973a) and van Damme (1991). The main difference is that we cannot define $\Phi$ globally (see the discussion of Harsanyi's 1973a approach following Theorem 1). Instead, we analyze the system of equations $\Phi(\bar{\sigma}, \bar{U})=0$ locally and apply this construction to a countable set of games and equilibria. Haller and Lagunoff (2000) also use local arguments to show the local finiteness of the equilibrium set. We, in contrast, use local arguments only to dispense with equilibria that are not quasistrict.

Having disposed of all games that have some equilibrium that fails to be quasistrict, we turn to games that have equilibria that all are quasistrict. Within this class, we restrict attention to completely mixed equilibria. The second lemma shows that for these equilibria, the Jacobian of $f$ with respect to the pair $(\sigma, U)$ has full rank. Its proof is similar to that of Claim 1 and exploits Lemma 1, and the diagonal structure of the Jacobian.

To state the lemma, we define the set of completely mixed profiles in $\Sigma^{\epsilon}$ as

$$
\widetilde{\Sigma}=\left\{\sigma \in \Sigma^{\epsilon} \mid \sigma_{i}\left(a_{i}, s\right)>0 \text { for all } i \in I, a_{i} \in A_{i}(s), \text { and } s \in S\right\} .
$$

Lemma 3. If $\sigma \in \widetilde{\Sigma}$, then $\partial f(\sigma, U) / \partial(\sigma, U)$ has full rank $\sum_{i \in I} \sum_{s \in S}\left|A_{i}(s)\right|$.

Proof. In matrix notation, $\sigma_{i}\left(a_{i}, s\right)\left(\mathcal{U}_{i}\left(a_{i}, s, \sigma\right)-\mathcal{U}_{i}\left(a_{i}^{s}, s, \sigma\right)\right)$ in equation (3.3) can be written as $\sigma_{i}\left(a_{i}, s\right)\left(\mathcal{P}_{a_{i}, \sigma_{-i}}^{s}-\mathcal{P}_{a_{i}^{s}, \sigma_{-i}}^{s}\right)\left(\mathbb{I}_{\sum_{s \in S}|A(s)|}-\delta_{i} Q \mathcal{P}_{\sigma}\right)^{-1} U_{i}$. For all $a_{i} \in A_{i}(s) \backslash\left\{a_{i}^{s}\right\}$ and $s \in S$, define the $\left(a_{i}, s\right)$ row of $P_{i}^{*}(\sigma) \in \mathbb{R}^{\sum_{s \in S}\left(\left|A_{i}(s)\right|-1\right) \times \sum_{s \in S}|A(s)|}$ by $\sigma_{i}\left(a_{i}, s\right) \times$ $\left(\mathcal{P}_{a_{i}, \sigma_{-i}}^{s}-\mathcal{P}_{a_{i}^{s}, \sigma_{-i}}^{s}\right.$. The components of $f$ associated with player $i$ can now be written as

$$
f_{i}(\sigma, U)=\left(\begin{array}{c}
\sum_{a_{i} \in A_{i}} \sigma_{i}\left(a_{i}, s_{1}\right)-1 \\
\vdots \\
\sum_{a_{i} \in A_{i}} \sigma_{i}\left(a_{i}, s_{|S|}\right)-1 \\
P_{i}^{*}(\sigma)\left(\mathbb{I}_{\sum_{s \in S}|A(s)|}-\delta_{i} Q \mathcal{P}_{\sigma}\right)^{-1} U_{i}
\end{array}\right) .
$$


The derivative of the first $|S|$ components of $f_{i}$ with respect to $\sigma_{i}$ takes the form

$$
\left(\begin{array}{ccc|ccc|c|cc|c}
\multicolumn{3}{c}{\sigma_{i}\left(\cdot, s_{1}\right)} & \multicolumn{3}{|c|}{\sigma_{i}\left(\cdot, s_{2}\right)} & \cdots & \multicolumn{3}{|c}{\sigma_{i}\left(\cdot, s_{|S|}\right)} \\
\hline 1 & \cdots & 1 & 0 & \cdots & 0 & \cdots & 0 & \cdots & 0 \\
0 & \cdots & 0 & 1 & \cdots & 1 & \cdots & 0 & \cdots & 0 \\
\vdots & & \vdots & \vdots & & \vdots & & \vdots & & \vdots \\
0 & \cdots & 0 & 0 & \cdots & 0 & \cdots & 1 & \cdots & 1
\end{array}\right) .
$$

This matrix, denoted by $X_{i}$, has full rank $|S|$. The Jacobian of the first $|S|$ components of $f_{i}$ with respect to $\left(\sigma_{-i}, U\right)$ is $0 \in \mathbb{R}^{|S| \times\left(\sum_{j \neq i}\left|A_{j}\right||S|+|I| \sum_{s \in S}|A(s)|\right)}$.

Next consider the components of $f_{i}$ associated with $a_{i} \neq a_{i}^{s}$. The Jacobian of those components with respect to $U$ takes the form

$$
\left(\begin{array}{c|c|c|c|c|c|c|c|c|}
U_{1}\left|U_{2}\right| & \cdots & U_{i-1} \mid & U_{i} & \left|U_{i+1}\right| & \cdots & U_{|I|} \\
\hline 0 & 0 & \cdots & 0 & \mid P_{i}^{*}(\sigma)\left(\mathbb{I}_{\sum_{s \in S}|A(s)|}-\delta_{i} Q \mathcal{P}_{\sigma}\right)^{-1} & 0 & \cdots & 0
\end{array}\right) .
$$

The matrix $P_{i}^{*}(\sigma)$ has full rank $\sum_{s \in S}\left(\left|A_{i}(s)\right|-1\right)$. To see this, note that for all pairs $\left(a_{i}, s\right)$ with $a_{i} \in A_{i}(s) \backslash\left\{a_{i}^{s}\right\}$, the $\left(a_{i}, s\right)$ row of $P_{i}^{*}(\sigma)$ contains a zero in all those components $\left(a^{\prime}, s^{\prime}\right)$ where either $s^{\prime} \neq s$ or $a_{i}$ is not contained in $a^{\prime}$ (by this we mean that there is no $a_{-i}$ such that $\left.\left(a_{i}, a_{-i}\right)=a^{\prime}\right)$. The $\left(a_{i}, s\right)$ row also contains a nonzero term in some component $\left(a^{\prime}, s\right)$ where $a^{\prime}$ contains $a_{i}$; indeed, $\sum_{a^{\prime} \text { contains } a_{i}} \sigma_{-i}\left(a^{\prime} \backslash a_{i}, s\right)=\sum_{a_{-i}} \sigma_{-i}\left(a_{-i}, s\right)=$ 1 , where, given $a^{\prime}=\left(a_{i}, a_{-i}\right)$, we write $a^{\prime} \backslash a_{i}=a_{-i}$. Since $P_{i}^{*}(\sigma)$ has full rank, so does the matrix $Z_{i}=P_{i}^{*}(\sigma)\left(\mathbb{I}_{\sum_{s \in S}|A(s)|}-\delta_{i} Q \mathcal{P}_{\sigma}\right)^{-1}$ as a consequence of Lemma 1.

We now see that, up to permutations of rows, the Jacobian of $f$ with respect to the pair $(\sigma, U)$ takes the form

$$
\frac{\partial f(\sigma, U)}{\partial(\sigma, U)}=\left(\begin{array}{c|c|c|c|c|c|c|c}
\sigma_{1} & \sigma_{2} & \cdots & \sigma_{|I|} & U_{1} & U_{2} & \cdots & U_{|I|} \\
\hline X_{1} & 0 & 0 & 0 & 0 & 0 & \cdots & 0 \\
0 & X_{2} & 0 & 0 & 0 & 0 & \cdots & 0 \\
\vdots & \vdots & \ddots & \vdots & \vdots & \vdots & & \vdots \\
0 & 0 & 0 & X_{|I|} & 0 & 0 & \cdots & 0 \\
\hline & & & & Z_{1} & 0 & \cdots & 0 \\
& & & & 0 & Z_{2} & \cdots & 0 \\
Y_{1} & Y_{2} & \cdots & Y_{|I|} & \vdots & \vdots & \ddots & \vdots \\
& & & & 0 & 0 & \cdots & Z_{|I|}
\end{array}\right),
$$

where $Y_{i} \in \mathbb{R}^{\sum_{i \in I} \sum_{s \in S}\left(\left|A_{i}(s)\right|-1\right) \times \sum_{s \in S}\left|A_{i}(s)\right|}$. This permits us to deduce that $\partial f(\sigma, U) /$ $\partial(\sigma, U)$ has full rank $\sum_{i \in I} \sum_{s \in S}\left|A_{i}(s)\right|$.

Lemma 3 implies a version of the structure theorem.

Corollary 3. The equilibrium graph has the same dimension as the space of games. 
Corollary 3 generalizes an observation made by Govindan and Wilson (2001) for normal form games to dynamic stochastic games. ${ }^{8}$ This corollary and the transversality theorem almost immediately yield Haller and Lagunoff's (2000) finiteness result in Corollary 1 . Note that to obtain a finiteness result it is enough to restrict attention to completely mixed equilibria; therefore, there is no need for Lemma 2. Our analysis also provides a finiteness proof for normal form games from a different perspective than the proof based on the theory of semialgebraic sets in Govindan and Wilson (2001). ${ }^{9}$

\subsubsection{Proof of Theorem 1}

We employ the following result from differential topology.

Theorem 3 (Transversality theorem). Let $\mathcal{O} \subseteq \mathbb{R}^{n}$ be open and let $L: \mathcal{O} \times \mathbb{R}^{s} \rightarrow \mathbb{R}^{n}$ be continuously differentiable. Assume that the Jacobian $\partial L(x, y) / \partial(x, y)$ has rank $n$ for all $(x, y) \in \mathcal{O} \times \mathbb{R}^{s}$ such that $L(x, y)=0$. Then, for almost all $\bar{y} \in \mathbb{R}^{s}$, the Jacobian $\partial L(x, \bar{y}) / \partial x$ has rank $n$ for all $x \in \mathcal{O}$ such that $L(x, \bar{y})=0$.

The transversality theorem is a generalization of the well known Sard theorem. See Mas-Colell et al. (1995) for an intuitive discussion and applications in economics, and see Abraham and Robbin (1967) and Guillemin and Pollack (1974) for further results and technical details.

We are now ready to prove Theorem 1 . Denote by $I$ the set of all games having some irregular equilibrium. Then

$$
\bar{I}=\bigcup_{C^{*} \subseteq B^{*}} I\left(B^{*}, C^{*}\right) .
$$

Since there exists only a finite number of sets $B^{*}$ and $C^{*}$ such that $C^{*} \subseteq B^{*}$, it is enough to show that $\lambda\left(I\left(B^{*}, C^{*}\right)\right)=0$ for all such sets. If $B^{*} \neq C^{*}$, this follows from Lemma 2 .

Suppose $B^{*}=C^{*}$ and consider the submatrix $\bar{J}(\sigma)$ obtained from $J(\sigma)=$ $\partial f(\sigma, U) / \partial \sigma$ by crossing out all rows and columns that correspond to components $\left(a_{i}, s\right)$ with $a_{i} \notin B_{i}^{*}(s)$. As shown in the proof of Proposition 1,

$$
|\operatorname{det}(J(\sigma))|=|\operatorname{det}(\bar{J}(\sigma))|\left|\prod_{i \in I} \prod_{s \in S} \prod_{a_{i} \notin C_{i}(\sigma, s)}\left[\mathcal{U}_{i}\left(a_{i}, s, \sigma\right)-\mathcal{U}_{i}\left(a_{i}^{s}, s, \sigma\right)\right]\right| .
$$

Since $\mathcal{U}_{i}\left(a_{i}, s, \sigma\right)-\mathcal{U}_{i}\left(a_{i}^{s}, s, \sigma\right)<0$ for $a_{i} \notin C_{i}(\sigma, s), J(\sigma)$ has full rank if and only if $\bar{J}(\sigma)$ does. The submatrix $\bar{J}(\sigma)$ is itself the Jacobian of a completely mixed equilibrium. Without loss of generality, we can therefore assume that $B^{*}(s)=C^{*}(s)=A(s)$ for all $s \in S$.

\footnotetext{
${ }^{8}$ Govindan and Wilson (2009) recently strengthened Corollary 3 by extending Kohlberg and Mertens' (1986) structure theorem to dynamic stochastic games.

${ }^{9}$ Note, however, that extending Govindan and Wilson's (2001) tools to dynamic stochastic games requires establishing the "semialgebraicity" of $f$.
} 
Then

$$
\begin{aligned}
& I\left(B^{*}, C^{*}\right) \subseteq\left\{U \in \mathbb{R}^{|I| \sum_{s \in S}|A(s)|} \mid \text { there exists } \sigma \in \widetilde{\Sigma}\right. \text { such that } \\
& \left.\qquad f(\sigma, U)=0 \text { and } \frac{\partial f(\sigma, U)}{\partial \sigma} \text { is singular }\right\} .
\end{aligned}
$$

From Lemma 3, $\partial f(\sigma, U) / \partial(\sigma, U)$ has full rank for all pairs $(\sigma, U) \in \widetilde{\Sigma} \times \mathbb{R}^{|I| \sum_{s \in S}|A(s)|}$. The transversality theorem therefore implies that for almost all games $U \in \mathbb{R}^{|I| \sum_{s \in S}|A(s)|}$, $\partial f(\sigma, U) / \partial \sigma$ has full rank whenever $f(\sigma, U)=0$.

\subsection{Proving Theorem 2}

To prove Theorem 2, we proceed as follows. In Section 7.2.1, we first derive a system of nonlinear equations that characterizes the equilibrium distributions of a perturbed dynamic stochastic game. In Section 7.2.2, we then exploit a result from algebraic topology to ensure that there exists a solution to this system and, moreover, that this solution is close enough to the regular equilibrium $\bar{\sigma}$ of the original (unperturbed) game.

\subsubsection{Alternative characterization}

We derive a system of nonlinear equations that characterizes the equilibrium distributions of a perturbed dynamic stochastic game. This, in effect, amounts to providing an alternative characterization of a Bayesian Markov perfect equilibrium. See Hotz and Miller (1993) and Aguirregabiria and Mira (2007) for similar derivations.

Continuation values. Consider a dynamic stochastic game with perturbations $\left(\mu_{i}(\cdot ; s)\right)_{i \in I, s \in S}$ and equilibrium strategy profile $b$. Let $\sigma^{b}$ be the corresponding consistent distribution profile. Then $\bar{V}_{i}: S \rightarrow \mathbb{R}$, the equilibrium value function for player $i$, is the solution the Bellman equation

$$
\begin{aligned}
\bar{V}_{i}(s)=u_{i}\left(\sigma^{b}(\cdot, s), s\right)+\sum_{a_{i} \in A_{i}(s)} \int_{\left\{\eta_{i} \mid b_{i}\left(s, \eta_{i}\right)=a_{i}\right\}} \eta_{i}\left(a_{i},\right. & \left.\sigma_{-i}^{b}(\cdot, s)\right) d \mu_{i}\left(\eta_{i} ; s\right) \\
& +\delta_{i} \sum_{s^{\prime} \in S} \bar{V}_{i}\left(s^{\prime}\right) q\left(s^{\prime} ; \sigma^{b}(\cdot, s), s\right) .
\end{aligned}
$$

The first and the third terms on the right hand side of equation (7.1) depend on $b$ only indirectly through $\sigma^{b}$. Proposition 1 in Hotz and Miller (1993) ensures that

$$
\sum_{a_{i} \in A_{i}(s)} \int_{\left\{\eta_{i} \mid b_{i}\left(s, \eta_{i}\right)=a_{i}\right\}} \eta_{i}\left(a_{i}, \sigma_{-i}^{b}(\cdot, s)\right) d \mu_{i}\left(\eta_{i} ; s\right)=e_{i}\left(\sigma^{b}, s\right),
$$

where $e_{i}\left(\sigma^{b}, s\right)$ is the expected value of the private shock given optimizing behavior. Hence, the second term in equation (7.1) is seen to also depend on $b$ only indirectly through $\sigma^{b}$. See Aguirregabiria and Mira (2002) for further discussion. 
Importantly, $e_{i}(\sigma, s)$ is well defined even if $\sigma \in \Sigma$ is not an equilibrium distribution. Moreover, $e_{i}(\sigma, s)$ is a continuous function of $\sigma \in \Sigma$. We note that for all $s \in S$ the range of $e_{i}(\cdot, s): \Sigma \rightarrow \mathbb{R}$ is contained in the interval $\left[-\gamma_{i}(s), \gamma_{i}(s)\right]$, where

$$
\gamma_{i}(s)=\left|A_{i}(s)\right| \int \max _{a \in A(s)}\left\{\left|\eta_{i}(a)\right|\right\} d \mu_{i}\left(\eta_{i} ; s\right) .
$$

According to Tietze's extension theorem (Royden 1968), it is therefore possible to extend $e_{i}(\cdot, s)$ to $\bar{\Sigma}^{\epsilon}$, the closure of $\Sigma^{\epsilon}$, in a continuous manner such that its range is contained in the interval $\left[-\gamma_{i}(s), \gamma_{i}(s)\right]$. Slightly abusing notation, we denote the extended function by $e_{i}(\cdot, s): \overline{\Sigma^{\epsilon}} \rightarrow \mathbb{R}$.

Using this construction, the value of continued play, given an arbitrary profile $\sigma \in \bar{\Sigma}^{\epsilon}$, is

$$
\bar{V}_{i}(\cdot, \sigma)=\left(\mathbb{I}_{|S|}-\delta_{i} \mathcal{P}_{\sigma} Q\right)^{-1}\left(\mathcal{P}_{\sigma} U_{i}+e_{i}(\sigma)\right),
$$

where the $s$ component of $e_{i}(\sigma) \in \mathbb{R}^{|S|}$ is given by $e_{i}(\sigma, s)$. We interpret $\bar{V}_{i}(s, \sigma)$ as the expected net present value of the stream of payoffs to player $i$ if the dynamic system is currently in state $s$ and play is according to $\sigma$. Note that the formula above reduces to equation (2.3) if $\mu_{i}(\{0\} ; s)=1$ for all $s$.

Equilibrium distributions. Fix $\sigma \in \bar{\Sigma} \epsilon$ and let $\bar{V}_{i}(\cdot, \sigma)$ be the corresponding value of continuation play. Define the best reply of player $i$ in state $s$ as

$$
\begin{aligned}
b_{i}^{\sigma}\left(s, \eta^{i}\right)=\arg \max _{a_{i} \in A_{i}(s)} u_{i}\left(a_{i}, \sigma_{-i}(\cdot, s), s\right)+\eta_{i}\left(a_{i},\right. & \left.\sigma_{-i}(\cdot, s)\right) \\
& +\delta_{i} \sum_{s^{\prime} \in S} \bar{V}_{i}\left(s^{\prime}, \sigma\right) q\left(s^{\prime} ; a_{i}, \sigma_{-i}(\cdot, s), s\right) .
\end{aligned}
$$

The number $b_{i}^{\sigma}\left(s, \eta^{i}\right)$ is the best reply of player $i$ if the current state is $s$, his private shock is $\eta_{i}$, his rivals play according to $\sigma_{-i}$, and player $i$ plays according to $\sigma_{i}$ from the subsequent period on.

For $a_{i} \in A_{i}(s)$, define the $\left(i, a_{i}, s\right)$ component of the function $g: \bar{\Sigma}^{\epsilon} \rightarrow \Sigma$ by

$$
g_{i, a_{i}, s}(\sigma)=\int_{\left\{\eta_{i} \mid b_{i}^{\sigma}\left(s, \eta_{i}\right)=a_{i}\right\}} d \mu_{i}\left(\eta_{i} ; s\right) .
$$

The number $g_{i, a_{i}, s}(\sigma)$ is the probability that the best reply of player $i$ in state $s$ is $a_{i}$. The following lemma characterizes the equilibrium distributions of the dynamic stochastic game with perturbations $\left(\mu_{i}(\cdot ; s)\right)_{i \in I}$ as fixed points of $g$.

Lemma 4. A profile $\sigma \in \bar{\Sigma}^{\epsilon}$ is an equilibrium distribution if and only if $g(\sigma)=\sigma$.

This lemma is standard up to the fact that the domain of $g$ is not $\Sigma$ but $\bar{\Sigma}^{\epsilon}$. It follows because the range of $g$ is contained in $\Sigma$, so that a fixed point of $g$ must belong to $\Sigma$. 
Finally, for $a_{i} \in A_{i}(s)$, define the $\left(i, a_{i}, s\right)$ component of the function $h: \bar{\Sigma} \epsilon \rightarrow$ $\mathbb{R}^{\sum_{i \in I} \sum_{s \in S}\left|A_{i}(s)\right|}$ by

$$
h_{i, a_{i}, s}(\sigma)= \begin{cases}\sum_{a_{i} \in A_{i}} \sigma_{i}\left(a_{i}, s\right)-1 & \text { if } a_{i}=a_{i}^{s} \\ g_{i, a_{i}, s}(\sigma)-\sigma_{i}\left(a_{i}, s\right) & \text { if } a_{i} \neq a_{i}^{s},\end{cases}
$$

where $a_{i}^{s}$ is the reference action for player $i$ in state $s$ as used in the construction of the function of $f$ for the equilibrium $\bar{\sigma}$ of the unperturbed game $U$. Since $g$ is continuous in $\sigma$, so is $h$. It is not hard to see that $h(\sigma)=0$ if and only if $g(\sigma)=\sigma$, so the problem of finding an equilibrium distribution reduces to finding a zero of $h$.

\subsubsection{Proof of Theorem 2}

We employ the following result from algebraic topology.

Proposition 5 (Govindan et al. 2003). Suppose that $O$ is a bounded, open set in $\mathbb{R}^{m}$ and $h, f: \bar{O} \rightarrow \mathbb{R}^{m}$ are continuous, where $\bar{O}$ denotes the closure of $O$. Further, suppose that $f$ is continuously differentiable on $O$, that $x_{0}$ is the only zero of $f$ in $O$, and that the Jacobian of $f$ at $x_{0}$ has full rank. If, for all $t \in[0,1]$, the function th $+(1-t) f$ has no zero on the boundary of $O$, then $h$ has a zero in $O$.

The equilibrium $\bar{\sigma}$ of the unperturbed game $U$ is a zero of $f$. We have also constructed $h$ so that a zero of $h$ is an equilibrium distribution of the perturbed game. In what follows, we use Proposition 5 to establish the existence of a Bayesian Markov perfect equilibrium of the perturbed game.

Since the equilibrium $\bar{\sigma}$ of the unperturbed game $U$ is regular, the argument developed in the proof of Proposition 2 shows that there exists an open set $O \subseteq \Sigma^{\epsilon}$ that satisfies the following conditions (referred to hereafter as C1-C5).

C1. $\bar{\sigma} \in O$.

C2. For all $\sigma \in O,\|\bar{\sigma}-\sigma\|<\bar{\epsilon}$.

C3. $\bar{\sigma}$ is the only zero of $f(\cdot, U)$ in $O$.

C4. For all $i \in I, s \in S$, and $a_{i} \in A_{i}(s)$, if $\bar{\sigma}_{i}\left(a_{i}, s\right)>0$, then $\sigma_{i}\left(a_{i}, s\right)>0$ for all $\sigma \in O$.

C5. For all $i \in I$, all $s \in S$, and $a_{i} \in A_{i}(s)$, if $\mathcal{U}_{i}\left(a_{i}, s, \bar{\sigma}\right)-\mathcal{U}_{i}\left(a_{i}^{s}, s, \bar{\sigma}\right)<0$, then $\mathcal{U}_{i}\left(a_{i}, s, \sigma\right)-\mathcal{U}_{i}\left(a_{i}^{s}, s, \sigma\right)<0$ for all $\sigma \in O$.

Consider the sequence of probability distributions of private shocks $\left(\mu_{i}^{n}(\cdot ; s)\right)_{n \in \mathbb{N}}$. For all $i \in I$, use $\left(\mu_{i}^{n}(\cdot ; s)\right)_{s \in S}$ to construct $e_{i}^{n}, \bar{V}_{i}^{n}, g^{n}$, and $h^{n}$ as detailed in Section 7.2.1. To prove Theorem 2, it suffices to find a zero of $h^{n}$ in $O$ for all large enough $n$. Such a zero is an equilibrium distribution of the dynamic stochastic game with perturbations $\left(\mu_{i}^{n}(\cdot ; s)\right)_{i \in I, s \in S}$ and it is within a distance at most $\bar{\epsilon}$ of $\bar{\sigma}$ due to C2. As a consequence of Proposition 5, the following lemma yields the desired result. 
Lemma 5. For all large enough $n$ and all $t \in[0,1]$, the function $t h^{n}+(1-t) f(\cdot, U)$ has no zero on the boundary of $O$.

Proof. Suppose not. Consider a sequence $\left(t^{n}\right)_{n \in \mathbb{N}}$ converging to $\bar{t} \in[0,1]$ and a sequence $\left(\sigma^{n}\right)_{n \in \mathbb{N}}$ contained in the boundary of $O$, converging to $\hat{\sigma}$, such that $\sigma^{n}$ is a zero of $t^{n} h^{n}+\left(1-t^{n}\right) f(\cdot, U)$. We state and prove three preliminary claims.

Claim 3. If $\mathcal{U}_{i}\left(a_{i}, s, \hat{\sigma}\right)-\mathcal{U}_{i}\left(a_{i}^{s}, s, \hat{\sigma}\right)<0$, then $g_{i, a_{i}, s}^{n}\left(\sigma^{n}\right) \rightarrow 0$.

Proof. For $s \in S, a_{i}^{\prime} \in A_{i}(s)$, and $\sigma \in \Sigma^{\epsilon}$, define

$$
\mathcal{U}_{i}^{n}\left(a_{i}^{\prime}, s, \sigma\right)=u_{i}\left(a_{i}^{\prime}, \sigma_{-i}(\cdot, s), s\right)+\delta_{i} \sum_{s^{\prime} \in S} \bar{V}_{i}^{n}\left(s^{\prime}, \sigma\right) q\left(s^{\prime} ; a_{i}^{\prime}, \sigma_{-i}(\cdot, s), s\right)
$$

Note that

$$
\bar{V}_{i}^{n}\left(\cdot, \sigma^{n}\right)=\left(\mathbb{I}_{|S|}-\delta_{i} \mathcal{P}_{\sigma^{n}} Q\right)^{-1}\left(\mathcal{P}_{\sigma^{n}} U_{i}+e_{i}^{n}\left(\sigma^{n}\right)\right) \quad \rightarrow \quad\left(\mathbb{I}_{|S|}-\delta_{i} \mathcal{P}_{\sigma} Q\right)^{-1} \mathcal{P}_{\sigma} U_{i}=V_{i}(\cdot, \sigma)
$$

because the range of $e_{i}^{n}(\cdot, s)$ is contained in $\left[-\gamma_{i}^{n}(s), \gamma_{i}^{n}(s)\right]$, where

$$
\gamma^{n}(s)=\left|A_{i}(s)\right| \int\left\{\max _{a \in A(s)}\left|\eta_{i}(a)\right|\right\} d \mu_{i}^{n}\left(\eta_{i} ; s\right) \rightarrow 0 .
$$

It thus follows that $\mathcal{U}_{i}^{n}\left(a_{i}^{\prime}, s, \sigma^{n}\right) \rightarrow \mathcal{U}_{i}\left(a_{i}^{\prime}, s, \hat{\sigma}\right)$ for all $s \in S$ and $a_{i}^{\prime} \in A_{i}(s)$. Consequently, there exists $\psi>0$ such that for all large enough $n$,

$$
\mathcal{U}_{i}^{n}\left(a_{i}, s, \sigma^{n}\right)-\mathcal{U}_{i}^{n}\left(a_{i}^{s}, s, \sigma^{n}\right)<-\psi
$$

By the definition of $g$ in equation (7.2),

$$
\begin{aligned}
& g_{i, a_{i}, s}^{n}\left(\sigma^{n}\right) \\
& \quad \leq \int_{\left\{\eta_{i} \in \mathbb{R}^{|A(s)|} \mid \eta_{i}\left(a_{i}, \sigma_{-i}^{n}(\cdot, s)\right)-\eta_{i}\left(a_{i}^{s}, \sigma_{-i}^{n}(\cdot, s)\right) \geq \mathcal{U}_{i}^{n}\left(a_{i}^{s}, s, \sigma^{n}\right)-\mathcal{U}_{i}^{n}\left(a_{i}, s, \sigma^{n}\right)\right\}} d \mu_{i}^{n}\left(\eta_{i} ; s\right) \\
& \quad \leq \int_{\left\{\eta_{i} \in \mathbb{R}^{|A(s)|}|| \eta_{i}\left(a_{i}, \sigma_{-i}^{n}(\cdot, s)\right)-\eta_{i}\left(a_{i}^{s}, \sigma_{-i}^{n}(\cdot, s)\right) \mid \geq \psi\right\}} d \mu_{i}^{n}\left(\eta_{i} ; s\right) \\
& \quad \leq \int_{\left\{\eta_{i} \in \mathbb{R}^{|A(s)|}|| \eta_{i}\left(a_{i}, \sigma_{-i}^{n}(\cdot, s)\right)-\eta_{i}\left(a_{i}^{s}, \sigma_{-i}^{n}(\cdot, s)\right) \mid \geq \psi\right\}} \frac{\left|\eta_{i}\left(a_{i}, \sigma_{-i}^{n}(\cdot, s)\right)-\eta_{i}\left(a_{i}^{s}, \sigma_{-i}^{n}(\cdot, s)\right)\right|}{\psi} d \mu_{i}^{n}\left(\eta_{i} ; s\right) \\
& \quad \leq \frac{2}{\psi} \int\left\{\max _{a \in A(s)}\left|\eta_{i}(a)\right|\right\} d \mu_{i}^{n}\left(\eta_{i} ; s\right) \\
& \quad \rightarrow 0 .
\end{aligned}
$$

Claim 4. If $\mathcal{U}_{i}\left(a_{i}, s, \hat{\sigma}\right)-\mathcal{U}_{i}\left(a_{i}^{s}, s, \hat{\sigma}\right)<0$, then $\hat{\sigma}_{i}\left(a_{i}, s\right)=0$. 
Proof. From Claim 3, $g_{i, a_{i}, s}^{n}\left(\sigma^{n}\right) \rightarrow 0$. Therefore,

$$
\begin{aligned}
0 & =\lim _{n \rightarrow \infty} t^{n} h_{i, a_{i}, s}^{n}\left(\sigma^{n}\right)+\left(1-t^{n}\right) f_{i, a_{i}, s}\left(\sigma^{n}, U\right) \\
& =\hat{t}\left(-\hat{\sigma}_{i}\left(a_{i}, s\right)\right)+(1-\hat{t}) f_{i, a_{i}, s}(\hat{\sigma}, U) \\
& =-\hat{\sigma}_{i}\left(a_{i}, s\right)\left(\hat{t}+(1-\hat{t})\left(\mathcal{U}_{i}\left(a_{i}^{s}, s, \hat{\sigma}\right)-\mathcal{U}_{i}\left(a_{i}, s, \hat{\sigma}\right)\right)\right) .
\end{aligned}
$$

It follows that $\hat{\sigma}_{i}\left(a_{i}, s\right)=0$.

Claim 5. For all $i \in I$ and $s \in S, \hat{\sigma}_{i}(\cdot, s)$ is a probability distribution. In addition, there exist $i \in I, a_{i} \in A_{i}(s) \backslash\left\{a_{i}^{s}\right\}$, and $s \in S$ such that $f_{i, a_{i}, s}(\hat{\sigma}, U) \neq 0$.

Proof. If $\hat{\sigma}_{i}\left(a_{i}, s\right)<0$, then C4 implies that $\bar{\sigma}_{i}\left(a_{i}, s\right)=0$. Since $\bar{\sigma}$ is quasistrict, $\mathcal{U}_{i}\left(a_{i}, s, \bar{\sigma}\right)-\mathcal{U}_{i}\left(a_{i}^{s}, s, \bar{\sigma}\right)<0$ and, from C5, $\mathcal{U}_{i}\left(a_{i}, s, \hat{\sigma}\right)-\mathcal{U}_{i}\left(a_{i}^{s}, s, \hat{\sigma}\right)<0$. Claim 4 shows that $\hat{\sigma}_{i}\left(a_{i}, s\right)=0$, a contradiction. Therefore, $\hat{\sigma}_{i}\left(a_{i}, s\right) \geq 0$ for all $i \in I, s \in s$, and $a_{i} \in A_{i}(s)$. Further, because $h_{i, a_{i}^{s}, s}^{n} \equiv f_{i, a_{i}^{s}, s}$, we deduce that $f_{i, a_{i}^{s}, s}\left(\sigma^{n}, U\right)=0$ for all $n$, so $\hat{\sigma}_{i}(\cdot, s)$ is a well defined probability distribution over actions. From C3, $\hat{\sigma}$ cannot be a zero of $f(\cdot, U)$. So there must exist $i \in I, a_{i} \in A_{i}(s) \backslash\left\{a_{i}^{S}\right\}$, and $s \in S$ such that $f_{i, a_{i}, s}(\hat{\sigma}, U) \neq 0 . \triangleleft$

With these claims in hand, we are ready to complete the proof of Lemma 5. Fix $i \in I, a_{i} \in A_{i}(s) \backslash\left\{a_{i}^{s}\right\}$, and $s \in S$ as in Claim 5 for the rest of the proof. Note that $a_{i}^{s}$ cannot belong to $B_{i}(\hat{\sigma}, s)$. Indeed, if it did, then $\mathcal{U}_{i}\left(a_{i}, s, \hat{\sigma}\right)-\mathcal{U}_{i}\left(a_{i}^{s}, s, \hat{\sigma}\right) \leq 0$ and, from Claim $4, f_{i, a_{i}, s}(\hat{\sigma}, U)=0$, contradicting the definition of $a_{i}$ (Claim 5). Since $\bar{\sigma}$ is quasistrict, $\bar{\sigma}_{i}\left(a_{i}^{\prime}, s\right)>0$ for all $a_{i}^{\prime} \in B_{i}(\bar{\sigma}, s)$; this together with $\mathrm{C} 4$ implies that $\hat{\sigma}_{i}\left(a_{i}^{\prime}, s\right)>0$ for all $a_{i}^{\prime} \in B_{i}(\bar{\sigma}, s)$. Statement C5 implies that $B_{i}(\hat{\sigma}, s) \subseteq B_{i}(\bar{\sigma}, s)$ and, therefore, $\hat{\sigma}_{i}\left(a_{i}^{\prime}, s\right)>0$ for all $a_{i}^{\prime} \in B_{i}(\hat{\sigma}, s)$. Consequently,

$$
\sum_{a_{i}^{\prime} \in B_{i}(\hat{\sigma}, s)} f_{i, a_{i}^{\prime}, s}\left(\sigma^{n}, U\right)>0 .
$$

For $a_{i}^{\prime} \notin B_{i}(\hat{\sigma}, s), g_{i, a_{i}^{\prime}, s}^{n}\left(\sigma^{n}\right) \rightarrow 0$, so that $\sum_{a_{i}^{\prime} \in B_{i}(\hat{\sigma}, s)} g_{i, a_{i}^{\prime}, s}^{n}\left(\sigma^{n}\right) \rightarrow 1$. Because $\hat{\sigma}_{i}\left(a_{i}^{s}, s\right)>0$ and $\hat{\sigma}(\cdot, s)$ is a probability distribution, $\sum_{a_{i}^{\prime} \in B_{i}(\hat{\sigma}, s)} \hat{\sigma}_{i}\left(a_{i}^{\prime}, s\right)<1$. Therefore,

$$
\sum_{a_{i}^{\prime} \in B_{i}(\hat{\sigma}, s)} h_{i, a_{i}^{\prime}, s}^{n}\left(\sigma^{n}\right)>0
$$

for large enough $n$. But equations (7.4) and (7.5) imply that $t^{n} h^{n}+\left(1-t^{n}\right) f(\cdot, U)$ is not zero at $\sigma^{n}$, a contradiction. This completes the proof of Lemma 5.

\section{Appendix}

In this appendix, we detail the construction of $\Sigma^{\epsilon}$. We then provide the proof of supporting results. 


\section{A.1 Construction of $\Sigma^{\epsilon}$}

We construct $\Sigma^{\epsilon}$ as follows. Note that, for all $\sigma \in \Sigma, \mathbb{I}_{|S|}-\delta_{i} \mathcal{P}_{\sigma} Q$ and $\mathbb{I}_{\sum_{s \in S}|A(s)|}-\delta_{i} Q \mathcal{P}_{\sigma}$ are invertible. Indeed, $\mathcal{P}_{\sigma} Q$ and $Q \mathcal{P}_{\sigma}$ are stochastic matrices so that $\mathbb{I}_{|S|}-\delta_{i} \mathcal{P}_{\sigma} Q$ and $\mathbb{I}_{\sum_{s \in S}|A(s)|}-\delta_{i} Q \mathcal{P}_{\sigma}$ have strictly dominant diagonals. Therefore, for all $\bar{\sigma} \in \Sigma$, we can find $\epsilon_{\bar{\sigma}}>0$ such that $\mathbb{I}_{|S|}-\delta_{i} \mathcal{P}_{\sigma} Q$ and $\mathbb{I}_{\sum_{s \in S}|A(s)|}-\delta_{i} Q \mathcal{P}_{\sigma}$ are invertible for all $\sigma \in \mathbb{R}^{\sum_{i \in I} \sum_{s \in S}\left|A_{i}(s)\right|}$ satisfying $\|\bar{\sigma}-\sigma\|<\epsilon_{\bar{\sigma}}$. Since $\Sigma$ is compact, we can take a finite covering $\left(B\left(\bar{\sigma}^{j}, \epsilon_{\bar{\sigma}^{j}}\right)\right)_{j \in J}$ of $\Sigma$. Define $\Sigma^{\epsilon}$ to be open such that its closure, denoted $\bar{\Sigma}^{\epsilon}$, is contained in the open set $\bigcup_{j \in J} B\left(\bar{\sigma}^{j}, \epsilon_{\bar{\sigma}^{j}}\right)$.

\section{A.2 Omitted proof}

Proof of Proposition 4. For large enough $n, C_{i}(\bar{\sigma}, s) \subseteq C_{i}\left(\sigma^{n}, s\right)$ for all $i \in I$ and $s \in S$. By definition of $g$ in equation (7.2), for any $a_{i} \in C_{i}\left(\sigma^{n}, s\right), g_{i, a_{i}, s}^{n}\left(\sigma^{n}\right)=\sigma_{i}^{n}\left(a_{i}, s\right)>0$. Therefore, there exists a set $R_{i, s}^{n} \subseteq \mathbb{R}^{|A(s)|}$ such that $\mu_{i}^{n}\left(R_{i, s}^{n} ; s\right)>0$ and, for all $\eta_{i} \in R_{i, s}^{n}$ and $a_{i}^{\prime} \in A_{i}(s)$,

$$
\mathcal{U}_{i}^{n}\left(a_{i}, s, \sigma^{n}\right)-\mathcal{U}_{i}^{n}\left(a_{i}^{\prime}, s, \sigma^{n}\right)>\eta_{i}\left(a_{i}^{\prime}, \sigma_{-i}^{n}(\cdot, s)\right)-\eta_{i}\left(a_{i}, \sigma_{-i}^{n}(\cdot, s)\right),
$$

where $\mathcal{U}_{i}^{n}$ is defined in equation (7.3). We can integrate out this inequality to deduce that, for all $a_{i}^{\prime} \in A_{i}(s)$,

$$
\begin{aligned}
\mathcal{U}_{i}^{n}\left(a_{i}, s, \sigma^{n}\right)-\mathcal{U}_{i}^{n}\left(a_{i}^{\prime}, s, \sigma^{n}\right) & \\
& >\frac{1}{\mu_{i}^{n}\left(R_{i, s}^{n} ; s\right)} \int_{\eta_{i} \in R_{i, s}^{n}} \eta_{i}\left(a_{i}^{\prime}, \sigma_{-i}^{n}(\cdot, s)\right)-\eta_{i}\left(a_{i}, \sigma_{-i}^{n}(\cdot, s)\right) d \mu_{i}^{n}\left(\eta_{i} ; s\right) .
\end{aligned}
$$

Letting $n \rightarrow \infty$, it follows that, for all $a_{i}^{\prime} \in A_{i}(s)$,

$$
\mathcal{U}_{i}\left(a_{i}, s, \sigma\right)-\mathcal{U}_{i}\left(a_{i}^{\prime}, s, \sigma\right) \geq 0 .
$$

We have therefore shown that for any $a_{i} \in C_{i}(\bar{\sigma}, s), a_{i} \in B_{i}(\bar{\sigma}, s)$. This proves the result.

\section{REFERENCES}

Abraham, Ralph and Joel W. Robbin (1967), Transversal Mappings and Flows. W. A. Benjamin, New York. [393]

Acemoglu, Daron and James A. Robinson (2001), "A theory of political transitions." American Economic Review, 91, 938-963. [369]

Aguirregabiria, Victor and Chun-Yu Ho (2008), "A dynamic oligopoly game of the US airline industry: Estimation and policy experiments." Working Paper 337, Department of Economics, University of Toronto. [386]

Aguirregabiria, Victor and Pedro Mira (2002), "Swapping the nested fixed point algorithm: A class of estimators for discrete Markov decision models." Econometrica, 70, 1519-1543. [394] 
Aguirregabiria, Victor and Pedro Mira (2007), "Sequential estimation of dynamic discrete games.” Econometrica, 75, 1-53. [371, 394]

Bajari, Patrick, C. Lanier Benkard, and Jonathan Levin (2007), "Estimating dynamic models of imperfect competition.” Econometrica, 75, 1331-1370. [371]

Bergemann, Dirk and Juuso Valimaki (1996), "Learning and strategic pricing." Econometrica, 64, 1125-1149. [369]

Bernheim, B. Douglas and Debraj Ray (1989), "Markov perfect equilibria in altruistic growth economies with production uncertainty." Journal of Economic Theory, 47, 195-202. [369]

Besanko, David, Ulrich Doraszelski, Yaroslav Kryukov, and Mark Satterthwaite (2010), "Learning-by-doing, organizational forgetting, and industry dynamics." Econometrica, 78, 453-508. [385]

Bhaskar, V. (1998), "Informational constraints and the overlapping generations model: Folk and anti-folk theorems.” Review of Economic Studies, 65, 135-149. [372, 379]

Bhaskar, V., George J. Mailath, and Stephen Morris (2007), "Purification in the infinitelyrepeated prisoners' dilemma.” Working Paper 07-024, Penn Institute for Economic Research. [372, 379]

Bhaskar, V., George Mailath, and Stephen Morris (2010), “A foundation for Markov equilibria in perfect information games." Unpublished paper, Department of Economics, Princeton University. [372, 379]

Borkovsky, Ron, Ulrich Doraszelski, and Yaroslav Kryukov (forthcoming), "A user's guide to solving dynamic stochastic games using the homotopy method." Operations Research. [385]

Doraszelski, Ulrich and Ariel Pakes (2007), "A framework for applied dynamic analysis in IO.” In Handbook of Industrial Organization, Volume 3 (Mark Armstrong and Robert H. Porter, eds.), 1887-1966, North-Holland, Amsterdam. [369, 373]

Doraszelski, Ulrich and Mark Satterthwaite (2010), “Computable Markov-perfect industry dynamics.” Rand Journal of Economics, 41, 215-243. [372, 377]

Ely, Jeffrey C. and Juuso Välimäki (2002), “A robust folk theorem for the prisoner's dilemma." Journal of Economic Theory, 102, 84-105. [379]

Ericson, Richard and Ariel Pakes (1995), "Markov-perfect industry dynamics: A framework for empirical work.” Review of Economic Studies, 62, 53-82. [369, 377]

Escobar, Juan F. (2008), "Existence of pure and behavior strategy stationary Markov equilibrium in dynamic stochastic games." Unpublished paper, Department of Economics, Stanford University. [387]

Fink, Arlington M. (1964), “Equilibrium in a stochastic $n$-person game.” Journal of Science of the Hiroshima University, Series A-I, 28, 89-93. [374] 
Fudenberg, Drew and Eric Maskin (1986), “The folk theorem in repeated games with discounting or with incomplete information.” Econometrica, 54, 533-554. [379]

Govindan, Srihari, Philip J. Reny, and Arthur J. Robson (2003), "A short proof of Harsanyi's purification theorem." Games and Economic Behavior, 45, 369-374. [372, 388, 396]

Govindan, Srihari and Robert Wilson (2001), "Direct proofs of generic finiteness of Nash equilibrium outcomes.” Econometrica, 69, 765-769. [393]

Govindan, Srihari and Robert Wilson (2009), "Global Newton method for stochastic games.” Journal of Economic Theory, 144, 414-421. [393]

Guillemin, Victor and Alan Pollack (1974), Differential Topology. Prentice Hall, Englewood Cliffs, New Jersey. [393]

Haller, Hans and Roger Lagunoff (2000), "Genericity and Markovian behavior in stochastic games.” Econometrica, 68, 1231-1248. [371, 375, 381, 382, 385, 388, 391, 393]

Harsanyi, J. C. (1973a), "Oddness of the number of equilibrium points: A new proof.” International Journal of Game Theory, 2, 235-250. [370, 371, 377, 380, 391]

Harsanyi, John C. (1973b), "Games with randomly disturbed payoffs: A new rationale for mixed-strategy equilibrium points.” International Journal of Game Theory, 2, 1-23. [370, 371, 377, 386, 387]

Herings, P. Jean-Jacques and Ronald J. A. P. Peeters (2004), "Stationary equilibria in stochastic games: Structure, selection, and computation.” Journal of Economic Theory, 118, 32-60. [382]

Horst, Ulrich (2005), "Stationary equilibria in discounted stochastic games with weakly interacting players.” Games and Economic Behavior, 51, 83-108. [370, 373]

Hotz, V. Joseph and Robert A. Miller (1993), “Conditional choice probabilities and the estimation of dynamic models.” Review of Economic Studies, 60, 497-529. [394]

Judd, Kenneth L. and Che-Lin Su (2008), "Constrained optimization approaches to estimation of structural models.” Unpublished paper, Hoover Institution, Stanford. [371]

Kalandrakis, Tasos (2006), "Regularity of pure strategy equilibrium points in a class of bargaining games.” Economic Theory, 28, 309-329. [371]

Kohlberg, Elon and Jean-François Mertens (1986), "On the strategic stability of equilibria." Econometrica, 54, 1003-1037. [393]

Kojima, Masakazu, Akira Okada, and S. Shindoh (1985), "Strongly stable equilibrium points of $n$-person noncooperative games." Mathematics of Operations Research, 10, 650-663. [370, 383]

Mailath, George J. and Stephen Morris (2002), "Repeated games with almost-public monitoring.” Journal of Economic Theory, 102, 189-228. [379] 
Mas-Colell, Andreu, Michael D. Whinston, and Jerry R. Green (1995), Microeconomic Theory. Oxford University Press, Oxford. [393]

Maskin, Eric and Jean Tirole (2001), "Markov perfect equilibrium, I: Observable actions.” Journal of Economic Theory, 100, 191-219. [371]

Mertens, Jean-François and T. Parthasarathy (1987), "Existence and characterization of Nash equilibria for discounted stochastic games.” Discussion Paper 8750, CORE, Louvain-la-Neuve. [370, 373]

Mertens, Jean-François and T. Parthasarathy (1991), "Nonzero-sum stochastic games.” In Stochastic Games and Related Topics: In Honor of Professor L. S. Shapley (T. E. S. Raghavan, T. Ferguson, T. Parthasarathy, and O. Vrieze, eds.), 145-148, Kluwer Academic Publishers, Dordrecht. [370]

Nowak, A. S. and T. E. S. Raghavan (1992), "Existence of stationary correlated equilibria with symmetric information for discounted stochastic games." Mathematics of Operations Research, 17, 519-526. [370, 373]

Pakes, Ariel, Michael Ostrovsky, and Steven Berry (2007), "Simple estimators for the parameters of discrete dynamic games (with entry/exit examples)." Rand Journal of Economics, 38, 373-399. [371]

Pesendorfer, Martin and Philipp Schmidt-Dengler (2008), "Asymptotic least squares estimators for dynamic games.” Review of Economic Studies, 75, 901-928. [371]

Phelan, Christopher and Andrzej Skrzypacz (2009), "Beliefs and private monitoring." Unpublished paper, Graduate School of Business, Stanford University. [379]

Ritzberger, Klaus (1994), "The theory of normal form games from the differentiable viewpoint.” International Journal of Game Theory, 23, 207-236. [382]

Royden, Halsey L. (1968), Real Analysis, second edition. Macmillan, New York. [395]

Shapley, Lloyd S. (1953), "Stochastic games.” Proceedings of the National Academy of Sciences of the United States of America, 39, 1095-1100. [369]

van Damme, Eric (1991), Stability and Perfection of Nash Equilibria, second edition. Springer, New York. [371, 377, 383, 391]

Wu, Wen-tsün and Jia-he Jiang (1962), "Essential equilibrium points of $n$-person noncooperative games.” Scientia Sinica, 11, 1307-1322. [370, 385]

Zangwill, Willard I. and C. B. Garcia (1981), Pathways to Solutions, Fixed Points, and Equilibria. Prentice Hall, Englewood Cliffs, New Jersey. [385]

Submitted 2009-9-21. Final version accepted 2010-3-15. Available online 2010-3-15. 